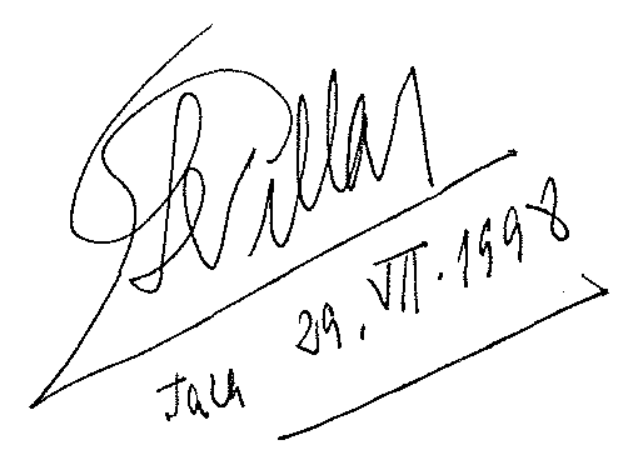

\title{
HEATHLAND VEGETATION OF THE NORTHERN-CENTRAL PART OF THE IBERIAN PENINSULA
}

\author{
Javier Loidi, Itziar García-Mijangos, Mercedes Herrera, Asun Berastegi \& Ainhoa \\ Darquistade
}

Department of Plant Biology and Ecology (Botany), Science Faculty, Basque Country University, Ap. 644, E-48080 Bilbao, Spain; fax +3444648500,E-mail gvploarj@lg.ehu.es

Keywords: Calluno-Ulicetea, Classification, Multivariate analysis, Ordination, Phytosociology, Syntaxonomy

\begin{abstract}
Heathland vegetation of northern Spain, included in the Calluno-Ulicetea, was studied using a set of 802 phytosociological relevés. The existing syntaxonomy has been tested and most of the types (associations and subassociations) fit satisfactorily with the observed groupings. Two main problems were encountered within the Ulex dominated communities of the Cantabrian fringe and the Castilian-Cantabrian heathland communities. Both groups of communities were subject to ordination in order to clarify relationships between them. For the former group, ordination suggests that three associations can be distinguished: the Ulici-Ericetum vagantis (lowlands up to the submontane belt), the Vaccinio-Ulicetum gallii for the communities of higher altitudes (montane belt) and the Ulici-Ericetum ciliaris (hygrophilous heathlands). The Castilian-Cantabrian heathlands show a variable Mediterranean influence and have a dispersed distribution due to lithological conditions. This results in the distinction of two new associations, viz. the Arctostaphylo crassifoliae-Daboecietum cantabricae (marly, water-retaining soils) and the Ericetum scopario-vagantis (sandy soils). A complete classification of the Calluno-Ulicetea in the studied area and short ecological and biogeographical diagnoses are given.
\end{abstract}

\section{INTRODUCTION}

Heathlands, dominated by ericaceous shrubs on acid soils, are one of the most characteristic vegetation types of Atlantic Europe. They are abundant in the Eurosiberian Region with a wet and oceanic temperate climate, extending to the northern part of the continent and including the British Isles. In the Mediterranean part, they are much less frequent, due to drier climatic conditions, and are present only in exceptionally rainy areas of the western and southwestern part of the Iberian Peninsula. The species richness of heath communities shows an inverse relationship to their abundance: the Ibero-Mediterranean communities bear a much richer flora than the northern ones, probably due to the absence of impoverishment during the recent Ice Age. Their development and proliferation have been traditionally related to man-induced fire and grazing. In most of the Atlantic regions of Europe, heathlands occupy large areas, particularly in mountains or on siliceous substrata (e.g. sandstone), considered unsuitable for arable use. Historically, they have been often subject to grazing and harvesting in the context of traditional land use of the Atlantic countries. The communities discussed are included in the Calluno-Ulicetea (for a comprehensive monography see Rivas-MarTínez 1979). From the biogeographical point of view, this heathland vegetation is distributed in the Atlantic and Mediterranean-Iberoatlantic Superprovinces, being also present in the rainy siliceous areas of NW Morocco. 
The aim of this work is to summarise and revise available information for the Calluno-Ulicetea in the central area of the northern fringe of the Iberian Peninsula (Basque Country, Navarra, La Rioja and the surrounding areas of Aragón, Cantabria and Castillia). The heathland communities of these territories have been studied in detail (GUINEA 1949, Braun-Blanquet 1967, Navarro 1986, Tarazona \& Zaldívar 1987, García-Mijangos 1994, TARAZONA 1984). The early classifications into associations and subassociations are at variance with our observations and we have therefore attempted a revision of all the relevé material available.

\section{MATERIAL AND METHODS}

A total of 802 phytosociological relevés were used. Most of them (691) are published or available in theses or unpublished reports. 111 new relevés were made for the purpose of this survey. For data processing the TABLAS program (QUINTANA 1993) was used to introduce the relevés into the database, to make the phytosociological tables and to transform them for later statistical treatment. The abundance-dominance data were transformed to absence/presence binary values using the TRAFOA program (FISCHER 1989).

Numerical classification with MULVA (WILDI 1991) and two detrended correspondence analysis (DCA) ordinations, using the CANOCO package (TER BRAAK 1988), were made. One of them was made with 285 relevés to clarify the syntaxonomical position of the communities from the Basque-Cantabrian Sector dominated by differents species of Ulex. The other one, with 175 relevés from the Mediterranean Region and the Alava-Navarran Subsector, attempted to clasify the syntaxonomical relationships of the Castilian-Cantabrian communities. Results are graphically displayed through CanoDraw (ŠMmAUER 1992).

Synthetic tables and syntaxonomy, constructed according to the principles of the BRAUN-BLANQUET (1964) approach, were constructed essentially by using our professional judgement, basical comparison of the literature and results of the ordinations.

Biogeographic and bioclimatic typologies follow RIVAS-MARTínEZ (1989), RIVAS-MARTínez et al. (1991) and LoIDI et al. (1994).

The nomenclature of plants follows CASTROVIEJo et al. (1986-1993). For taxa not included in the latter work, TUTIN et al. (1964-1980) was followed. Trinomial names of subspecies have been abbreviated into binomial ones in tables by dropping the middle (species) names.

\section{RESULTS}

\section{Basque-Cantabrian communities}

\section{Erica ciliaris heathlands}

These heathlands occur on humid soils at low altitudes (colline belt). GunNEA (1949) in his phytosociological work from the Basque Country made no distinction between those communities with $E$. ciliaris and those without, although a large set of relevés was presented. Later BRAUN-BLANQUET (1967) ascribed these communities to the Ulici-Ericetum ciliaris. Other authors (RIVAS-MARTíNEZ 1979, NAVARRO 1980, LoIDI 1983) considered this unit as a subassociation of other broadly-conceived associations. Finally, the original position of BRAUN-BLANQUET was accepted by RIVAS-MARTÍNEZ et al. (1991). The ordination represented in Fig. 2 shows a tendency for those relevés with Erica ciliaris to be grouped together and so we include them within the Ulici-Ericetum ciliaris. 


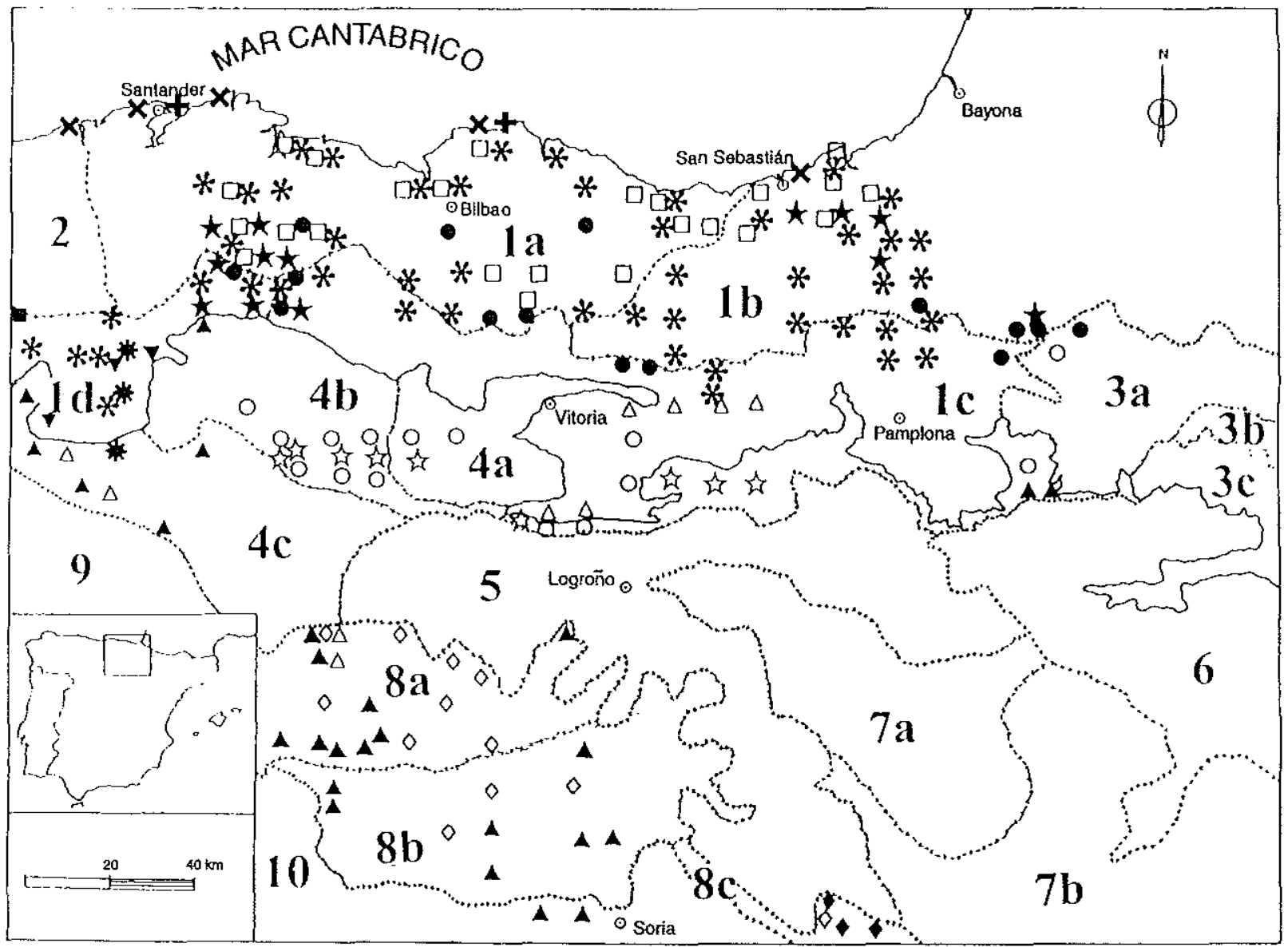

Fig. 1. Map of the surveyed area and its division into biogeographical units. Eurosiberian Region: Atlantic-Cantabrian Province: 1. Basque-Cantabrian Province, 1a. Santanderian-Biscaian Subsector, 1b. Eastern Basque Subsector, Ic. Alava-Navarran Subsector, 1d. Southern Cantabrian Subsector, 2. Galician-Asturian Sector; Pyrenean Province: 3. Central Pyrenean Sector, 3a. Western Pyrenean Subsector, 3b. High Pyrenean Subsector, 3c. Pre-Pyrenean Subsector. Mediterranean Region: Aragonese Province: 4. Castilian-Cantabrian Sector, 4a. Western Alavés Subsector, 4b. Mirandés Subsector, 4c. Páramos-Bureba Subsector, 5. Riojan Sector, 6. Somontano-Aragonés Sector, 7. Bardenas-Monegros Sector, 7a. Bardenero Subsector, 7b. Monegrino Subsector; Carpetano-Ibérico-Leonés Province: 8. Ibérico-Soriano Sector, 8a. Demandés Subsector, 8b. Urbionense Subsector, 8c. Moncayense Subsector; Castellano-Mastrazgo-Manchega Province: 9. Castellano-Duriense Sector, 10. Celtibérico-Alcareño Sector.

Location of the commented syntaxa: Ulicion minoris, Daboecienion: $\square$ Ulici-Ericetum ciliaris, Vaccinio myrtilli-Ulicetum gallii, $\star$ Erico tetralicis-Ulicetum gallii, * Ulici europaei-Ericetum vagantis, * Halimio umbellati-Daboecietum cantabricae, O Arctostaphylo crassifoliae-Daboecietum cantabricae, is Ericetum scopario-vagantis, Daboecio cantabricae-Ericetum aragonensis; Genistion micrantho-anglicae: $\mathbf{\Delta}$ Genisto anglicae-Ericetum vagantis, $\Delta$ Genisto anglicae-Daboecietum cantabricae, $\boldsymbol{\nabla}$ Euphorbio polygalifoliae-Ericetum tetralicis; Ericion umbellatae: $\bigcirc$ Genisto pilosae-Ericetum aragonensis, $\downarrow$ Calluno vulgaris-Genistetum occidentalis; Dactylido-Ulicenion maritimi: * Ulici humilis-Ericetum vagantis, \ Genisto occidentalis-Ulicetum maritimi.

\section{Ulex dominated heathlands}

The classification of the non-hygrophilous heathlands dominated by Ulex presented a complex situation. These communities are abundant on the Atlantic side of the Cantabrian Fringe, under wet, oceanic climatic conditions (Santanderian-Biscaian and Eastern Basque Subsectors, Fig. 1). Two species of Ulex, such as $U$. europaeus (ranging from the sea-level 


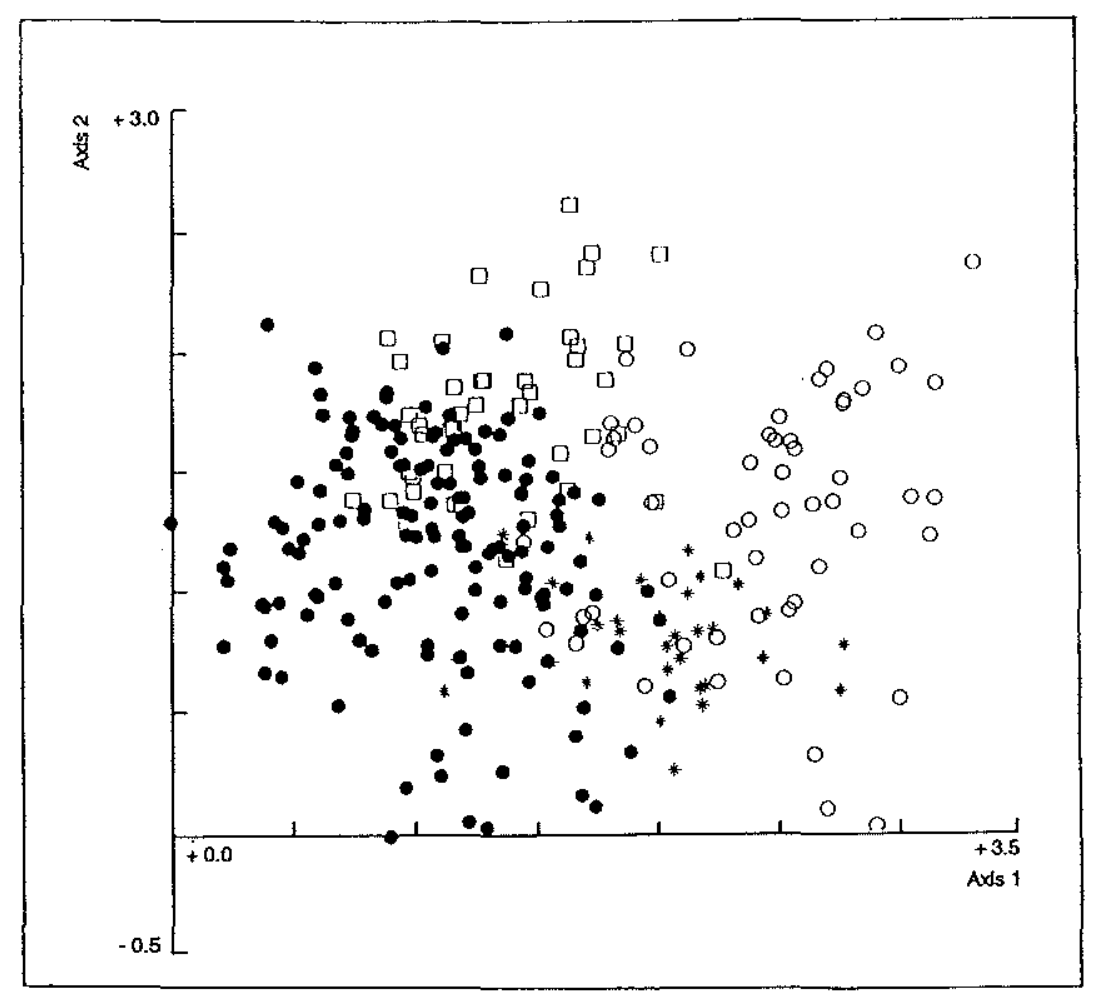

Fig. 2. DCA ordination of the reduced Daboecienion cantabricae data set. (Halimio umbellati-Daboecietum cantabricae, Arctostaphylo crassifoliae-Daboecietum cantabricae, Ericetum scopario-vagantis and Daboecio cantabricae-Ericetum aragonensis were discarded). Ulici-Ericetum ciliaris ( $\square$ ); Vaccinio myrtilli-Ulicetum gallii (*); Erico tetralicis-Ulicetum gallii $(\mathrm{O})$; Ulici europaei-Ericetum vagantis $(\bullet)$.

up to $700 \mathrm{~m}$ ) and $U$. gallii s.l. (found from low altitudes up to $1700 \mathrm{~m}$ ) occur here. In the colline and low-montane belts, both species co-exist, while in the middle and upper montane belts only $U$. gallii occurs. GUINEA (1949) validly published the Ulici-Ericetum vagantis based on data from the surroundings of Gernika at low altitudes. Recently, DÍAZ GonZÁLEZ \& FERNÁNDEZ PRIETO (1994) chose a type for this name from among the relevés of that table, but the relevé chosen only contained U. europaeus, and so the name selected had to be Ulici europaei-Ericetum vagantis (Rec. 10D). BRAUN-BLANQUET (1967), who did not mention the work of GUINEA, proposed the name Daboecio-Ulicetum europaei to incorporate all the gorse-rich heathlands of the Atlantic coast. He further subdivided the association into two subassociations, i.e. one for low altitudes (with U. europaeus) - lithodoretosum and one for high altitudes (rich in $U$. gallii) - ulicetosum gallii. RIVAS-MARTíneZ (1979) accepted the idea of splitting these heathlands into two subunits and suggested two associations based on the original subassociations of BRAUN-BLANQUET. It becomes clear, however, that the Daboecio-Ulicetum europaei lithodoretosum BR.-BL. 1967 is a later synonym of the Ulici-Ericetum vagantis GUINEA 1949, and thus the latter name prevails. There is a difficulty in the interpretation of the Daboecio-Ulicetum europaei ulicetosum gallii BR.-BL. 1967 in that the chosen type (by RIVAS-MARTínEZ 1979) contains no U. europaeus, despite this species being frequent in those places where the type relevé was made. We assume that $U$. europaeus remained probably unobserved by BRAUN-BLANQUET. This suggests that the ulicetosum gallii is also a synonym of the Ulici-Ericetum vagantis GUINEA 1949, because its nomenclatural type comes from an area in which both gorse species co-exist (a characteristic feature of the 


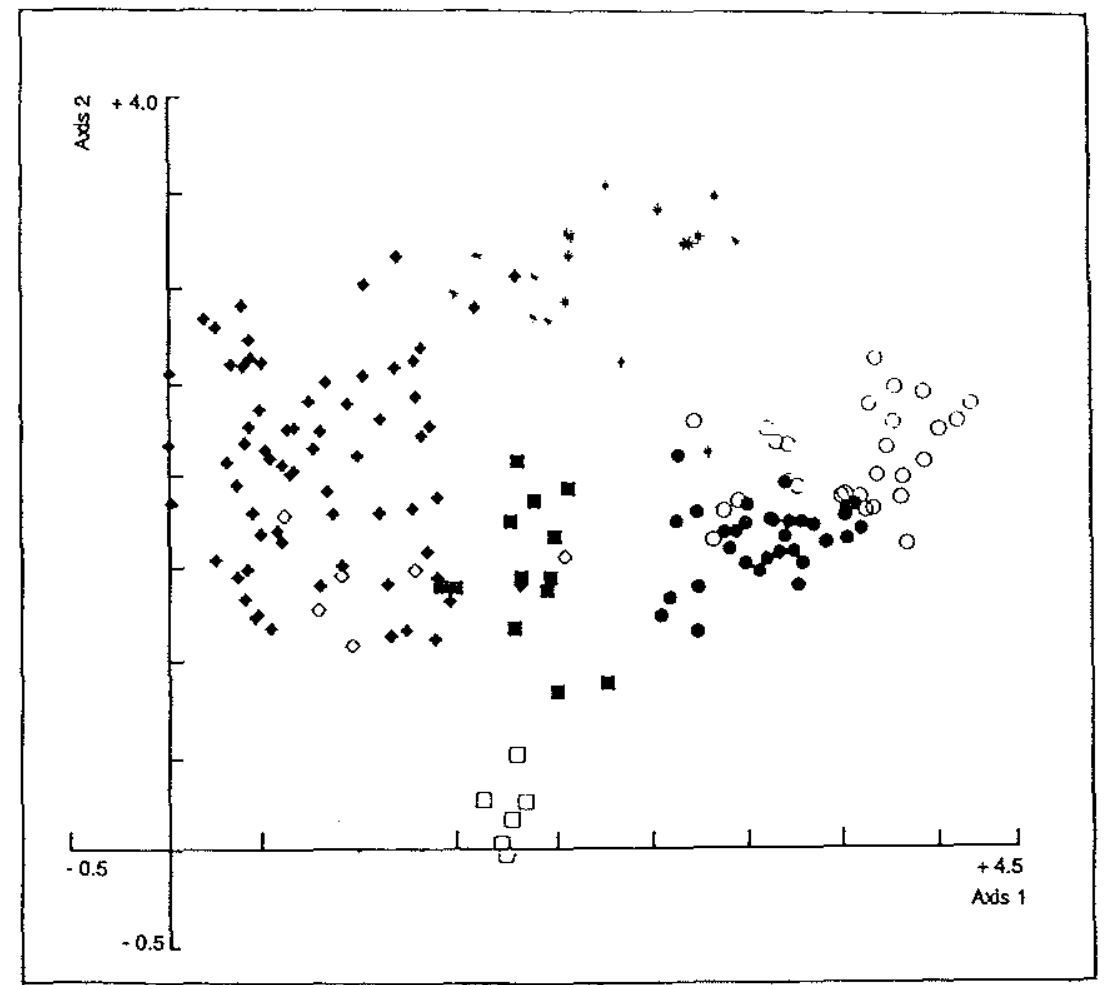

Fig. 3. DCA ordination of the non hygrophilic heathlands original from Alava-Navarran subsector and the Ibérico-Soriano and Castilian-Cantabrian sector. Erico tetralicis-Ulicetum gallii ( $\square$ ); Halimio umbellatae-Daboecietum cantabricae (1); Arctostaphylo crassifoliae-Daboecietum cantabricae (-); Ericetum scopario-vagantis $(\mathrm{O})$; Daboecio cantabricae-Ericetum aragonensis $(\diamond)$; Genisto pilosae-Ericetum aragonensis $(\downarrow)$; Calluno vulgaris-Genistetum occidentalis $(*)$.

Ulici-Ericetum). Thus, both units of BRAUN-BLANQUET belong to the same association but, consequently, the montane heathlands in which $U$. europaeus is absent need to be grouped within a unit of its own. The only validly published syntaxon available is the Daboecio-Ulicetum gallii vaccinietosum myrtilli (LOIDI 1983), a type which is clearly of montane distribution and contains no U. europaeus. For that reason, we accept two associations; a colline-submontane - Ulici europaei-Ericetum vagantis, and a montane - Vaccinio myrtilli-Ulicetum gallii. The diagram of Fig. 2 shows both associations separated on an ordination plane.

\section{Castilian-Cantabrian communities}

Fig. 3 shows the ordination of the relevés from the Alava-Navarran Subsector, Castilian-Cantabrian Sector and Ibérico-Soriano Sector. The Castilian-Cantabrian relevés form a distinct group from the others, corresponding to another area, and it is therefore necessary to propose a new syntaxonomical treatment for them.

The Castilian-Cantabrian Sector has a submediterranean climate and calcareous rocks dominate the geology of the region. These conditions are unfavourable for the development of heathlands. Nevertheless, suitable conditions can be found in some areas due to the high altitude, increased rainfall and patches of sandstone. As a result there is a significant representation of heathland vegetation in this Sector, although it is very fragmented and, due to the particular climatic and geological conditions, it is characterised by a high 
species-richness. In this sense, the situation is the opposite to that in the Basque-Cantabrian Sector in which heathlands occupy large areas but diversity is lower.

Two main associations can be recognised in this Sector: the Arctostaphylo crassifoliae-Daboecietum cantabricae (humid areas) and the Ericetum scopario-vagantis (dry areas), both showing a strong influence of the neighbouring stands of basiphilous scrub belonging to the Genistion occidentalis (Festuco hystricis-Ononidetea striatae).

The former community occurs in mountainous areas over marly soils with a high water-retention capacity. This association can be subdivided into three geographically distinct subassociations. The latter association, the Ericetum scopario-vagantis, contains fewer Calluno-Ulicetea species because it is found in somewhat drier habitats on patches of sand. The distribution of this community is patchy, consisting of many "islands" surrounded by the dominant Genistion occidentalis scrub.

Acknowledgements: This work was supported by a grant of the project GV 118.0124/94 sponsored by the Basque Country University and the Basque Government. Jennifer Duckworth and David Hoare corrected our English.

\section{REFERENCES}

BÁSCONES J.C. (1978): Relaciones suelo-vegetación en la Navarra húmeda del noroeste. Estudio florístico-ecológico. PhD. Thesis, Universidad de Navarra, Pamplona.

BRAUn-BlanqueT J. (1964): Pflanzensoziologie. Grundzüge der Vegetationskunde. Ed. 3. Springer, Wien.

BRAUN-BLANQUET J. (1967): Vegetationsskizzen aus dem Baskenland mit Ausblicken auf das weitere Ibero-Atlantikum. II. Teil. Vegetatio 14: 1-126.

Castroviejo S., Lainz M., López González G., Montserrat P., Muñoz Garmendia F., Paiva J. \& Villar L. (eds.) (1986-1993): Flora iberica 1-4. Real Jardin Botánico, C.S.I.C., Madrid.

CATALÁN P. (1987): Geobotánica de las cuencas Bidasoa-Urumea (NO de Navarra-NE de Guipuzcoa). Estudio ecológico de los suelos y de la vegetación de la cuenca de Artikutza (Navarra). PhD Thesis, Universidad del País Vasco, Bilbao.

DÍAZ GONZÁLEZT.E. \& FERNÁNDEZ PRIETO J.A. (1994): La vegetación de Asturias. Itinera Geobot. 8: 243-528.

FERNÁNDEZ PRIETO J.A. \& LOIDI J. (1984a): Datos sobre los brezales del Campoo. Lazaroa 5: 75-87.

FERNÁNDEZ PRIETO J.A. \& LOIDI J. (1984b): Estudio de las comunidades vegetales de los acantilados costeros de la comisa cantábrica. Doc. Phytosoc. N.S. 8: 185-218.

FisCHER H.S. (1989): Das Pflanzensoziologische Programm-System. Ed. Hagen S. Fischer, Erlangen.

GARCÍA-MiJAngos I. (1994): Flora y vegetación de los Montes Obarenes (Burgos). PhD Thesis, Universidad del País Vasco, Bilbao.

GUINEA E. (1949): Vizcaya y su paisaje vegetal (Geobotánica vizcaina). Junta de Cultura de Vizcaya, Bilbao.

HERRERA M. (1995): Estudio de la vegetación y flora vascular de la cuenca del río Asón (Cantabria). Guineana 1: $1-435$.

LOIDI J. (1983): Estudio de la flora y vegetación de las cuencas de los ríos Deva y Urola en la provincia de Guipuzcoa. PhD Thesis, Universidad Complutense de Madrid, Madrid.

LOIDI J., BERASTEGI A. \& GARCÍA-MIJANGOS I. (1996): Notas nomenclaturales sobre la vegetación del norte de la Península Ybérica, V. Lazaroa 17: 141-146.

LOIDI J., BIURRUN I. \& HERRERA M. (1997): La vegetación del centro-septentrional de España. Itinera Geobot. 9: $161-618$.

LOIDI J. \& FERNÁNDEZ PRIETO J.A. (1986): Datos sobre la biogeographía y la vegetación del sector Castellano-Cantábrico (España). Doc. Phytotaoc. N.S. 10: 323-362.

Loidi J., Herrera M., García-MiJangos I., Cervello A., Biurrun I. \& SiluváN F. (1992): Los ecosistemas forestales, preforestales y pascícolas de las comarcas de Ayala, Mena y Ordua: Tipificación, procesos de degradación, propuestas para su preservación, valoración naturalistica. Final Report, Universidad del País Vasco, Bilbao. 
L.OIDI J., Hizrrira M. \& Biurrun 1. (1994): Datos sobre la vegetación del País Vasco y zonas limítrofes (La vegetación del Parque Natural de Valderejo). Servicio Central de Publicaciones del Gobierno Vasco, Vitoria.

Midrano Morino L.M. (1994): Flora y vegetación de las Sierras de la Demanda y Cameros (La Rioja). PhD. Thesis, Universidad de Navarra, Pamplona.

MENDiola M.A. (1983): Estudios de flora y vegetación en La Rioja (Sierra Cebollera). Instituto de Estudios Riojanos, Logroo.

NAVARROC. (1980): Contribución al estudio de la flora y vegetación del Duranguesado y la Busturia (Vizcaya). PhD. Thesis, Universidad Complutense de Madrid, Madrid.

Navarro C. (1983): Datos sobre la vegetación de Vizcaya (País Vasco). Lazaroa 4: 119-127.

NAvArRo G. (1986): Vegetación y flora de las sierras de Urbión. Neila y Cabrejas. PhD. Thesis, Universidad Complutense de Madrid, Madrid.

Navarko G. (1989): Contribución al conocimiento de la vegetación del Moncayo. Opusc. Bot. Pharm. Complut. 5: 5-64.

ONAINDIA M. (1986): Ecología vegetal de las Encartaciones y macizo del Gorbea. Serv. Ed. Universidad del País Vasco, Bilbao.

PERALTA DE ANDRÉs F.J. (1992): Suelos y vegetación de la Sierra de Leyre. PhD. Thesis, Universidad de Navarra, Pamplona.

PERALTA DE ANDRÉS F.J. \& BÁSCONES J.C. (1997): Datos sobre los brezales con Genista anglica L. de las sierras meridionales de Álava y Navarra. Itinera Geobot. 10: 353-363.

Peralta de Andrés F.J., ÍÑIguez J. \& BÁscones J.C. (1989): Suelos y vegetatión de las Peñas de Aya (Navarra y Quipúzcoa). Anales Edafol. Agrobiol. 48: 499-522.

QUiNTANA A. (1993): Aplicación TABLAS. Ed. Alberto Quintana, Bilbao.

RIVAS-MARTÍNEZ S. (1962): Contribución al estudio fitosociológico de los hayedos españoles. Anales Inst. Bot. Cavanilles 20: 99-128.

RIVAS-MARTÍNEZ S. (1979): Brezales y jarales de Europa occidental (Revisión fitosociológica de las clases Calluno-Ulicetea y Cisto-Lavanduletea). Lazaroa 1: 5-127.

RIVAS-MARTINEZ S. (1989): Bioclimatic belts of West Europe. Relations between bioclimate and plant ecosytems. European School of climatology and natural hazards. Commission of the European Communities, Arles (Rhône).

Rivas-Martínez S., Díaz GonzÁlez T.E., Fernández Prieto J.A., LoIdi J. \& Penas A. (1984): La vegetación de la alta montaña cantábrica: Los Picos de Europa. Ed. Leonesas, León.

RIVAS-MARTÍNEZ S, , LOIDIJ., CANTÓ P., SANCHO L.G. \& SÁNCHEZ-MATA D. (1985): Datos sobre la vegetación del valle del río Bidasoa (España). Lazaroa 6: 127-150.

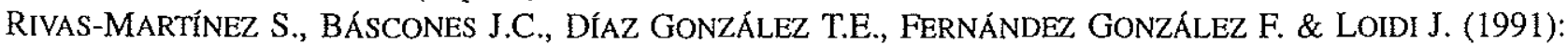
Vegetación del Pirineo occidental y Navarra. Itinera Geobot. 5: 5-456.

ŠMilAUER P. (1992): CanoDraw user guide. Version 3.0. Microcomputer Power, New York.

TARAZONA T. (1984): Estudio florístico, ecológico y fitosociológico de los matorrales del sector Ibérico-Soriano. Publ. Inst. Nac. Invest. Agrarias, Collect. Tesis Doct. 46: 1-355.

TARAZONA T. \& ZALDfVAR P. (1987): Nota sobre los brezales de la provincia de Burgos. Lazaroa 7: $351-362$.

TER BRAAK C.J.F. (1988): CANOCO - a Fortran program for canonical community ordination by [partial], [detrended], [canonical] correspondence analysis, principal component analysis and redundancy analysis (version 2.1.). Agricultural Mathematics Group, Ministry of Agriculture and Fisheries, Wageningen.

TUTIN T.G et al. (eds.) (1964-1980): Flora europaea 1-5. Cambridge University Press, Cambridge.

TÜXEN R. \& OBERDORFER E. (1958): Die Pflanzenwelt Spaniens II Teil. Eurosibirische Phanerogamen-Gesellschaften Spaniens. Veröff. Geobot. Inst. Rübel, Zürich 32: 1-328.

VAN DEN BERGHEN C. (1973): Les landes à Erica vagans de la Haute Soule (Pyrénées Atlantiques, France). Colloq. Phytosoc. 2: $91-96$.

WILDI O. (1991): MULVA-4, a processing environment for vegetation analysis. In: FEOLi E. \& ORLÓCI L. (eds.), Handbook of Vegetation Science 11: Computer assisted vegetation analysis, Kluwer Academic Publishers, Dordrecht, pp. 403-406.

Encl. Appendix pp. 266-281 


\section{APPENDIX}

\section{Syntaxonomical synopsis}

\section{Calluno-Ulicetea BR.-BL. et TÜXEN ex KLIKA et HADAČ 1944}

This class comprises scrub of acid soils with raw humus dominated by ericaceous shrubs, especially by heather, common in regions of high precipitation in W Europe as well as $\mathrm{N}$ Morocco. In the Mediterranean high precipitation is needed to compensate for summer-drought. The Calluno-Ulicetea are found from the sea-level up to the lower limit of the subalpine-oromediterranean belts.

Character-taxa: Calluna vulgaris, Carex pilulifera, Erica tetralix and Genista pilosa.

\section{Ulicetalia minoris QUANTIN 1935}

Syn.: Calluno-Ulicetalia (QUANTIN 1935) TUXXN 1937; Ulicetalia BR.-BL. ex ROTHMALER 1954; Erico-Ulicetalia BR.-BL. et al. 1964.

Heathlands of Atlantic, Mediterranean-Iberoatlantic and western north-African (Tingitania) distribution, in which some species of Ulex may become dominant.

Character-taxa: Agrostis curtisii, Erica australis subsp. aragonensis, E. ciliaris, E. cinerea, E. vagans, Euphorbia polygalifolia subsp. polygalifolia, Genistella tridentata, Halimium alyssoides, Lithodora prostrata, Scorzonera humilis, Simethis mattiazzi, Xolantha tuberaria and Viola lactea.

\section{Ulicion minoris MALCUIT 1929}

Syn.: Ulicion minoris DUVIGNEAUd 1944 (art. 22).

Atlantic communities of heath and gorses (Ulex).

Character-taxa: Cirsium filipendulum, Erica ciliaris, Pseudarrhenatherum longifolium, Serratula tinctoria, Ulex europaeus and $U$. gallii.

\section{Daboecienion cantabricae DUPONT ex RIVAS-MARTÍNEZ 1979}

Syn.: Daboecion cantabricae DUPONT 1975 nom. inval. (art. 2b, 8), Daboecienion cantabricae RIVAS-MARTÍNEZ in RIVAS-MARTÍNEZ et al. 1991.

Cantabrian communities.

Character-taxa: Daboecia cantabrica, Laserpitium prutenicum subsp. dufourianum.

\section{Ulici-Ericetum ciliaris BR.-BL. 1967}

Lectotypus: BRAUN-BLANQUET (1967: Tab. 25, rel. 4). Lectum: LoIDI et al. (1996).

Syn.: Daboecio-Ulicetum gallii ericetosum ciliaris RIVAS-MARTínEZ et al. in C. NAVARRo 1982.

Wet to hygromorphic soils originating on substrata of low permeability; colline, submontane; humid to hyperhumid; Basque-Cantabrian.

ericetosum vagantis (RIVAS-MARTíNEZ 1979) LOIDI et al. 1996

Type is the same as in the association.

Syn.: Cirsio filipenduli-Ericetum ciliaris BR.-BL. et al. 1964 ericetosum vagantis RIVAS-MARTINEZ 1979; Daboecio-Ulicetum gallii ericetosum ciliaris RIVAS-MARTínEZ et al. in C. NAVARRo 1982 p. p. excl. typus). ericetosum tetralicis LorDl et al. 1996

Lectotypus: BRAUN-BLANQUET (1967: Tab. 25, rel. 12).

Syn.: Daboecio-Ulicetum gallii ericetosum ciliaris RIVAS-MARTíNEZ et al. in C. NAVARRO 1982 p.p. incl. typus. Strongly hygromorphic soils.

\section{Vaccinio myrtilli-Ulicetum gallii ass. nova}

Holotypus: LoIDI (1983: Tab. 35, rel. 9).

Incl.: Daboecio-Ulicetum gallii vaccinietosum myrtilli LoIDI 1983; Daboecio-Ulicetum europaei ulicetosum gallii BR.-BL. 1967 p. min. p. excl. typus; Daboecio-Ulicetum cantabrici genistetosum pilosae RIVAS-MARTÍNEZ et al. 1991.

Montane (and colline in some continental valleys of the Cantabrian Mts.); humid-hyperhumid; Biogeographic units: Basque-Cantabrian, Ovetense, Central-eastern Orocantabrian.

Erico tetralicis-Ulicetum gallii LOIDI et al. in HERRERA 1995

Holotypus: TARAZONA \& ZALDIVAR (1987: Tab. 1, rel. 7). 
Incl.: Daboecio-Ulicetum gallii ericetosum tetralicis TARAZONA et ZALDÍVAR 1987.

Montane; hyperhumid; Basque-Cantabrian.

ulicetosum gallii LOIDI et al. in HERRERA 1995

Type is the same as in the association.

Mid-montane.

juniperetosum alpinae LOIDI et al. in HERRERA 1995

Holotypus: HERRERA (1995: Tab. 73, rel. 21).

High-montane.

\section{Ulici Europaei-Ericetum vagantis GUINEA 1949}

Lectotypus: GUINEA (1949: Tab. 10, rel. 5). Lectum: DÍAZ GONZÁLEZ \& FERNÁNDEZ PRIETO (1994: 392).

Syn.: Daboecio-Ulicetum europaei BR.-BL. 1967; incl. Daboecio-Ulicetum europaei BR.-BL. 1967

lithodoretostum BR.-BL. 1967; incl. Daboecio-Ulicettm europaei ulicetosum gallii BR.-BL. 1967 p.max.p. incl.

typus; Daboecio-Ulicetum gallii (BR.-BL. 1967) RIVAS-MARTíneZ 1979; Daboecio-Ulicetum cantabrici

(BR.-BL. 1967) RIVAS-MARTínEZ 1979 corr. RIVAS-MARTíNEZ et al. 1991 (art. 22).

Thermocolline, colline and submontane; humid-hyperhumid; Basque-Cantabrian, Ovetense and Northern

Galician-Asturian. Heathland with Ulex europaeus, U. gallii and Erica vagans.

ericetosum vagantis GUINEA 1949

Type is the same as in the association.

Throughout the range of the association.

genistetosum occidentalis (C. NAVARRO 1982) comb. nova

Holotypus: C. NAVARRo (1983: Tab. 25, rel. 1).

Basionym: Daboecio-Ulicetum gallii genistetosum occidentalis C. NAVARRO 1982.

Transitional unit to the Genistion occidentalis of contact with limestone and flysch.

Halimio umbellati-Daboecietum cantabricae FERNÁNDEZ PRIETO et LOIDI ex LOIDI et al. 1996

Holotypus: FERNÁNDEZ PRIETO \& LOIDI (1984: Tab. 2, rel. 3).

Syn.: Halimio umbellati-Daboecietum cantabricae FERNÁNDEZ PRIETO et LOIDI 1984 nom. inval. (art. 5). Supramediterranean, montane; subhumid-humid; Castilian-Cantabrian, Southern Cantabrian, Campurriano-

-Carrionés.

daboecietosum cantabricae FERNÁNDEZ PRIETO et LOIDI ex LOIDI et al. 1996

Type is the same as in the association.

Supramediterranean; Castilian-Cantabrian.

ulicetosum gallii FERNÁNDEZ PRIETO et LOIDI ex LOIDI et al. 1996

Holotypus: FERNÁNDEZ PRIETO \& LOIDI (1984: Tab. 1, rel. 12).

Syn.: Daboecio-Ulicetum gallii halimietosum umbellati FERNÁNDEZ PRIETO et LOIDI 1985 nom. inval. (art. 5).

Mesomontane; Southern Cantabrian.

genistetosum pilosae FERNÁNDEZ PRIETO et LOIDI 1984

Holotypus: FERNÁNDEZ PRIETO \& LOIDI (1984: Tab. 2, rel. 7).

High-montane; Altocampurriano.

genistelletosum tridentatae FERNÁNDEZ PRIETO et LOIDI 1984

Holotypus: FERNÁNDEZ PRIETO \& LOIDI (1984: Tab. 2, rel. 10).

Mesomontane; Campurriano-Carrionés.

Arctostaphylo crassifoliae-Daboecietum cantabricae ass. nova

Holotypus: La Molina (Burgos), 850 m a.s.l., $50 \mathrm{~m}^{2}$, 9.VIII.1991. Calluna vulgaris 5, Arctostaphylos uva-ursi subsp. crassifolia 3, Erica cinerea 3, Erica vagans 2, Cistus salviifolius 2, Pteridium aquilinum 2, Avenula sulcata 1, Potentilla montana 1, Genista occidentalis 1, Xolantha tuberaria 1, Dorycnium pentaphyllum 1, Avenula mirandana 1, Festuca rubra s.1. 1, Silene legionensis 1, Quercus pyrenaica juv. 1, Thesitum divaricatum 1, Geum sylvaticum +, Thymelaea ruizii + , Daboecia cantabrica + , Carex flacca + , Quercus faginea juv. + , Geranium sanguineum + , Quercus rotundifolia juv. + , Asperula cynanchica + , vicia cracca,+ Carex humilis +. Linum milleti + .

Castilian-Cantabrian, Alava-Navarran.

daboecietosum cantabricae subass. nova 
Type is the same as in the association.

Castilian-Cantabrian.

ulicetosum gallii TARAZONA \& ZALDÍVAR subass, nova

Holotypus: Tarazona \& Zaldívar (1987: Tab. 2, rel. 1).

Syn.: Daboecio-Ulicetum gallii arctostaphyletosum crassifoliace TARAZONA et ZALDÍVAR 1987 nom. inval. (art. 5)

Castilian-Cantabrian (Tesla)

ulicetosum etropaei subass. nova

Holotypus: Monte Cervera, Sierra de Cantabria (Alava), deep soil, $1230 \mathrm{~m}$ a.s.1., $100 \mathrm{~m}^{2}$, 25.VIII.1992.

Ulex europaeus 3, Erica vagans 3, Erica cinerea 2, Daboecia cantabrica 2, Avenula sulcata 1, Calluna valgaris

1, Potentilla erecta 1, Brachypodium rupestre 1, Genista pilosa 1, Danthonia decumbens + , Agrostis capillaris

+ , Serratula tinctoria + , Stachys officinalis + , Potentilla montana + , Helianthemum nummularium + , Rumex acetosa + , Solidago virgaurea + , Silene vulgaris + , Thymelaea ruizii + , Lotus sp. + .

Alava-Navarran.

Ericetum scopario-vagantis ass. nova

Holotypus: Bozoo (Burgos), $900 \mathrm{~m}$ a.s.1., $80 \mathrm{~m}^{2}$, 13.X.1990. Erica scoparia 4, Cistus salviifolius 2, Erica vagans 2, Juniperus oxycedrus 2, Erica cinerea 1, Calluna vulgaris 1, Potentilla montana 1, Dorycnitum pentaphyllum 1, Genista scorpius 1, Arctostaphylos crassifolia 1, Spiraea obovata 1, Filipendula vulgaris 1, Thymus vulgaris 1, Lavandula latifolia 1, Quercus rotundifolia juv. 1, Potentilla neumanniana 1, Geum sylvaticum 1, Thymelaea ruizii,+ Aphyllanthes monspeliensis,+ Teucrium chamaedrys,+ Helichrysum stoechas + , Helianthemum canum + .

Castilian-Cantabrian.

ericetosum scopariae subass. nova

Type is the same as in the association.

Sandy-loamy soils.

lavanduletosum pedunculatae GARCÍA-MIJANGOS subass. nova

Holotypus: DE ONAA A PINO DE BUREBA (Burgos), $600 \mathrm{~m}$ a.s.l., $30 \mathrm{~m}^{2}$, 17.VII.1991. Erica scoparia 5, Cistus salviifolius 1, Calluna vulgaris 1, Erica cinerea 1, Elaeoselinum gummiferum 1, Lavandula pedunculata 1, Thymus mastichina 1, Dorycnium pentaphyllum 1, Genista scorpius 1, Amelanchier ovalis 1, Quercus rotundifolia juv. 1, Brachypodium retusum 1, Phillyrea angustifolia 1, Quercus faginea juv. 1, Bupleurum rigidum 1, Helichrysum stoechas 1, Anthoxanthum odoratum 1, Erica vagans + , Sorbus domestica + , Juniperus oxycedrus + , Sanguisorba minor + , Quercus coccifera + , Centaurea ornata,+ Rubus ulmifolius,+ Anthericum liliago + , Carlina vulgaris,+ Malus sylvestris,+ Rosa corymbifera,+ Sedum forsteranum + .

Pure sandy soils.

Daboecio cantabricae-Ericetum aragonensis RIVAS-MARTÍNEZ in FERNÁNDEZ PRIETO et LOIDI 1984

Holotypus: FERNÁNDEZ PRIETO \& LOIDI (1984: Tab. 3, rel. 3)

Colline, montane; humid-hyperhumid; Orocantabrian.

Dactylido maritimae-Ulicenion maritimi (GÉHU 1975) suball. nova

Lectotypus: Ulici (europaei) maritimi-Ericetum cinereae (J.M. GÉHU 1962) J.M. et J. GÉHU 1973 (Colloque Intern. Végét. Landes Lille: 187). Lectum: hoc loco.

Bas.: Dactylido maritimae-Ulicion maritimi GÉHU 1975 (art. 27); Ulicenion maritimo-humilis (GÉHU 1975) RIVAS-MARTÍNEZ 1979

Cantabrian coastal heathlands with cushion-shaped gorses developed on coastal cliffs under influence of air-borne salt spray.

Character and differential taxa: Anthyllis vulneraria subsp. iberica (dif.), Armeria euskadiensis (dif.), Asparagus prostratus, Dactylis glomerata subsp. maritima (dif.), Ulex europaeus f. maritimus.

Ulici (gallii) humilis-Ericetum vagantis FERNÁNDEZ PRIETO et LOIDI 1984

Holotypus: FERNÁNDEZ PRIETO \& LOIDI (1984: Tab. 11, rel. 2).

Communities over hard limestones along the eastern half of the Cantabrian coasts.

ericetosum vagantis FERNÁNDEZ PRIETO et LOIDI 1984

Type is the same as in the association. 
Shallow soils.

ulicetosum maritimi FERNÁNDEZ PRIETO et LOIDI 1984

Holotypus: FERNÁNDEZ PRIETO \& LOIDI (1984: Tab. 11, rel. 10).

Deep soils.

Genisto occidentalis-Ulicetum maritimi C. NAVARRO 1983

Holotypus: C. NAVARRO (1983: Tab. 4, rel. 5).

Communities over easy-eroding rocks (e.g. flysch) along the eastern Cantabrian coasts.

\section{Genistion micrantho-anglicae RIVAS-MARTÍNEZ 1979}

Hygrophilous heathlands of Mediterranean-Ibero-Atlantic distribution developed on gley soils containing anmoor organic matter.

Character and differential taxa: Erica tetralix (dif.), Genista anglica and Genista micrantha.

Genisto anglicae-Ericetum vagantis RIVAS-MAR'rínez et TARAZONA in RIVAS-MARTÍNEZ 1979

Holotypus: RIVAS-MARTínez (1979: Tab. 19, rel. 2).

Supramediterranean; Ibérico-soriano, Castilian-Cantabrian.

ericetosum vagantis RIVAS-MARTÍNEZ et TARAZONA in RIVAS-MARTíNEZ 1979

Type is the same as in the association.

Gley but not permanently stagnant soils of the ibérico-soriano and castilian-cantabrian areas.

cistetosum laurifolii TARAZONA ex LOIDI et al. 1996

Holotypus: TARAZONA (1984: Tab. 4, rel. 16).

Syn.: Genisto anglicae-Ericetum vagantis cistetosum laurifolii TARAZONA 1984, nom. inval. (art. 5)

Southern flanks of the Sierra Mencilla; subhumid.

ericetosum tetralicis G. NAVARRO in LOIDI et al. 1997

Holotypus: LOIDI et al. (1997: 324).

Ibérico-Soriano; stagnant depressions.

ericetosum scopariae G. NAVARRO ined.

Southern Urbionense (Cuerda del Pozo dam).

arctostaphyletosum crassifoliae PERALTA et BÁsCONES 1997

Holotypus: PERALTA \& BÁSCONES (1997: Tab. 1, rel. 11).

Leire, Illón and Orba.

Genisto anglicae-Daboecietum cantabricae BÁsCONES et PERALTA in LOIDI et al. 1996

Holotypus: TARAZONA (1984: Tab. 4, rel. 21).

Incl.: Genisto anglicae-Ericetum vagantis daboecietosum cantabricae TARAZONA 1984, nom. inval. (art. 5)

Montane; humid-hyperhumid; Alava-Navarran, Southern Cantabrian and Castilian-Cantabrian.

daboecietosum cantabricae

Type is the same as in the association.

Alava-Navarran and Castilian-Cantabrian.

ulicetosum gallii LOIDI, FERNÁNDEZ PRIETO, A. BUENO et M. HERRERA subass. nova

Holotypus: From Las Machorras to the Pico de la Churra, Espinosa de los Monteros (Burgos), $900 \mathrm{~m}$ a.s.l., $100 \mathrm{~m}^{2}, 12$. VI.1990. Erica cinerea 3, Pteridium aquilinum 2, Ulex gallii 2, Genista micrantha 2, Erica vagans 2, Daboecia cantabrica 2, Pseudarrhenatherum longifolium 2, Calluna vulgaris 1, Agrostis curtisii 1, Potentilla erecta 1, Potentilla montana 1, Carex pilulifera 1, Lotus corniculatus 1, Polygala serpyllifolia 1, Hieracium pilosella 1, Anthoxanthum odoratum + , Digitalis parviflora + , Senecio sylvaticus + , Hypericum pulchrum + , Genista florida subsp. polygaliphylla,+ Jasione laevis + , Carlina vulgaris,+ Galium verum + , Stachys officinalis + , Arenaria montana + , Lithodora diffusa + , Erica arborea + .

Humid; Southem Cantabrian.

\section{Euphorbio polygalifoliae-Ericetum tetralicis FERNÁNDEZ PRIETO et LOIDI 1984}

Holotypus: FERNÁNDEZ PRIETO \& LOIDI (1984: Tab. 5, rel. 1).

Montane; Southern Cantabrian, Campurriano-Carrionés.

ericetosum tetralicis FERNÁNDEZ PRIETO et LOIDI 1984

Type is the same as in the association.

Campurriano-Carrionés. 
ericetosum vagantis l'ERNÁNDEZ PRIETO et LOIDI 1984

Holotypus: FERNÁNDEZ PRIETO \& LOIDI (1984: Tab. 5, rel. 3).

Southern Cantabrian.

Ericion umbellatae BR.-BL. et al. 1952

Mediterranean-Ibero-Atlantic heathlands; thermo-, meso- and supramediterranean; central-western and south-western parts of the Iberian Peninsula and NW Morocco.

Character-taxa: Genistella tridentata, Cistus psilosepalus, Halimitum ocymoides, H. umbellatum, Erica australis subsp. aragonensis and Luzula lactea.

Genisto pilosae-Ericetum aragonensis RIVAS-MARTÍNEZ 1979

Lectotypus: Rivas-MARTíneZ (1979: Tab. 11, rel. 6).

Supramediterranean; humid-hyperhumid; Ibérico-Soriano.

ericetosum aragonensis RIVAS-MARTíNEZ 1979

Type is the same as in the association.

cistetosum laurifolii TARAZONA ex LOIDI et al. 1996

Lectotypus: TARAZONA (1984: Tab. 3, rel. 12).

Syn.: Genisto pilosae-Ericetum aragonensis cistetosum laurifolii TARAZONA 1984 nom. inval. (art. 5).

genistetosum anglicae TARAZONA ex LOIDI et al. 1996

Transitional to hydromorphic soils.

Lectotypus: TARAZONA (1984: Tab. 3, rel. 15).

Syn.: Genisto pilosae-Ericetum aragonensis genistetosum anglicae TARAZONA 1984 nom. inval. (art. 5).

cytisetosum oromediterranei RIVAS-MARTÍNEZ $1979 \mathrm{corr}$.

Upper supramediterranean, transitional to the oromediterranean belt.

Holotypus: RIVAS-MARTÍNEZ (1979: Tab. 11, rel. 1).

Syn.: Genisto pilosae-Ericetum aragonensis cytisetosum purgantis RIVAS-MARTÍNEZ 1979 (art. 43)

daboecietosum cantabricae TARAZONA ex LOIDI et al. 1996

Demandés subsector, rainy areas of cantabrian influence.

Lectotypus: TARAZONA (1984: Tab. 3, rel. 23).

Syn.: Genisto pilosae-Ericetum aragonensis daboecietosum cantabricae TARAZONA 1984 nom. inval. (art. 5) pterospartetosum tridentati G. NAVARRO ined.

Upper supramediterranean (1500 to $1750 \mathrm{~m}$ ) in Urbión mountain. Represents an orophytic and somewhat continental version of the association.

Calluno vulgaris-Genistetum occidentalis RIVAS-MARTÍNEZ et G. NAVARRo in G. NAVARRo 1989

Holotypus: G. NAVARRo (1989: Tab. 14, rel. 6).

Supramediterranean; humid-hyperhumid; Moncayense.

genistetosum occidentalis RIVAS-MARTínEZ et G. NAVARRO in G. NAVARRO 1989

Type is the same as in the association.

Medium altitudinal range of the distribution of the association.

ericetosum aragonensis RIVAS-MARTínEZ et G. NAVARRO in G. NAVARRO 1989

Holotypus: G. NAVARRO (1989: Tab. 14, rel. 9).

High altitudinal range of the distribution of the association (Fagus sylvatica belt).

genistetosum micranthae RIVAS-MARTínEZ et G. NAVARRO in G. NAVARRO 1989

Holotypus: G. NAVARRO (1989: Tab. 14, rel. 12).

Wet soils.

lavanduletosum pedunculatae RIVAS-MARTÍNEZ et G. NAVARRO in G. NAVARRO 1989

Holotypus: G. NAVARRO (1989: Tab. 14, rel. 15).

Low altitudinal range of the distribution of the association (Quercus pyrenaica belt). 


\section{Table 1}

Ulici-Ericetum ciliaris (Daboecienion cantabricae) and Dactylido maritimae-Ulicenion maritimi. 1-16 Ulici-Ericetum ciliaris (1-9 ericetosum ciliaris, $10-16$ ericetosum tetralicis); 17-18 Ulici (gallii) humilis-Ericetum vagantis (17 ericetosum vagantis, 18 ulicetosum maritimi); 19-20 Genisto occidentalis-Ulicetum maritimi.

No. of column $1 \quad 2 \quad 3 \quad 4 \quad 5 \quad 6 \quad 7 \quad 8 \quad 91011121314151617181920$

No. of relevés

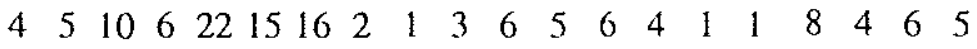

Differentials of the Ulici-Ericetum ciliaris (Daboecienion)

Erica ciliaris

Agrostis curtisii

Pseudarrhenatherum longifolium

Calluna vulgaris

Molinia caerulea s.1.

Ulex europaeus

Daboecia cantabrica

Danthonia decumbens

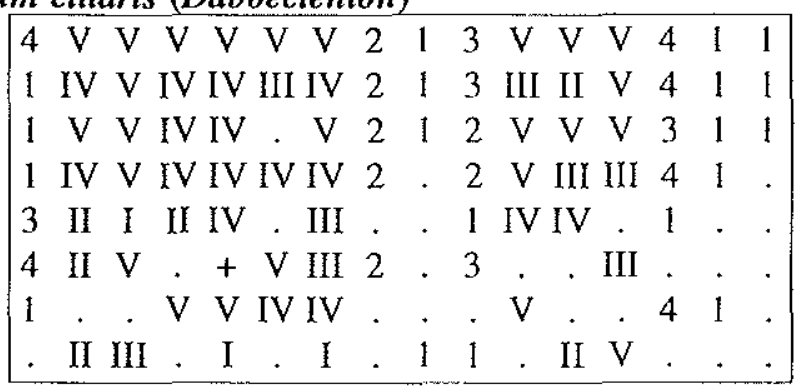

Differentials of the Ulici-Ericetum ciliaris ericetosum tetralicis

Erica tetralix

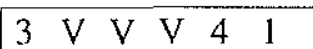

Differentials of the Dactylido maritimae-Ulicenion maritimi

Pulicaria odora

Daucus gummifer

Genista occidentalis

Leucanthemum crassifolium

Dactylis maritima

Lotus crassifolius

Plantago maritima

Koeleria glauca

Silene maritima

Ulex europaeus var. maritimus

Anthyllis iberica

\begin{tabular}{|cccc|}
\hline III & 2 & III &. \\
V & 1 & V & III \\
IV & 4 & IV & IV \\
V & 2 & IV &. \\
IV & 2 & III &. \\
III & 3 & III &. \\
III &. & III & II \\
II & 2 & I &. \\
I &. & I & II \\
. & 4 & V & V \\
. & 1 & IV &. \\
\hline
\end{tabular}

Character species of the high-rank units

Erica vagans

Erica cinerea

Potentilla erecta

Ulex gallii

Lithodora prostrata

Cirsium filipendulum

Serratula tinctoria

Avenula sulcata

Scorzonera humilis

Euphorbia angulata

Cistus salviifolius

Genista pilosa

\section{Companions}

Pteridium aquilinum

Brachypodium rupestre

Blechnum spicant

Scilla verna

Stachys officinalis

Salix atrocinerea

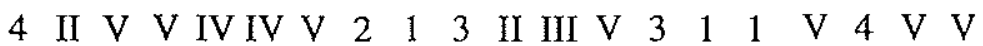

$\begin{array}{llllllllllllllll}4 & \text { IV IV IV V V V V } & 2 & 1 & 2 & V & \text { II } & V & 4 & 1 & 1 & \text { IV } & 2 & \text { I } & \text { II }\end{array}$

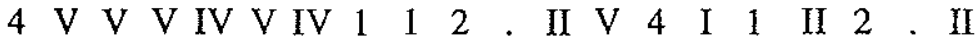

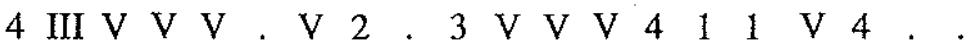

4 V IV III III I IV 1

3 II IV III II . II . . . . . II IV 2 . . . . . II II

. . IV II III + II 1 . . . . . . 1 .

. I . + . . . 1 . . . . . . .

. . ...$++ \cdot$. . V

. . III . . . . . . . . . III

. I . . . . . . . . . . . . V

I

$\begin{array}{lllllllllllllll}3 & \text { IV III V IV V V } & 1 & 1 & 3 & \text { IV } & \text { I } & \text { III } & 3 & 1 & 1 & \text { II } & 3 & \text {. II }\end{array}$

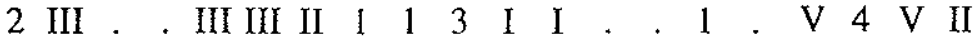

. I + III I IV I . . 1 II I II . . 1 . . . .

II III III I . . 1 . 2 . II $\mathrm{V}$. . . II 1 III .

I III. II IV I. . . . . I II . 1 . I I 1 I.

$\mathrm{I}+.+$ I II . . . I I I . . $1 \ldots .$.


No. of column 1234567891011121314151617181920

Agrostis capillaris

Schoenus nigricans

Picris hieracioides

Smilax aspera

Hypochoeris radicata

Asphodelus albus

Teucrium scorodonia

Carex flacca

Viola silvestris agg.

Quercus pyrenaica

Hypericum pulchrum

Polygala serpyllifolia

Potentilla montana

Lotus corniculatus

Galium saxatile

Festuca rubra agg.

Simethis mattiazzi

Centaurea nigra s.l.

Lithodora diffusa

Quercus robur

Carex binervis

Serratula seoanei

Frangula alnus

Deschampsia flexuosa

Plantago lanceolata

Gentiana pneumonanthe

Leontodon taraxacoides

Solidago virgaurea

Lobelia urens

Laserpitium dufourianum

Rubia peregrina

Rubus ulmifolius

Prunella hastifolia

\begin{tabular}{|c|c|c|c|c|c|c|c|c|c|c|c|c|c|c|c|}
\hline II III & & I & I & II & t & & & I & I & & 1 & & & & \\
\hline$I+$ & & I & + & + & 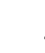 & & & IV & I & & & & V & & II \\
\hline I I & & + & $1+$ & + & . & & & & I & & & & II & & \\
\hline . + & & + & +1 & I & & & . & & & & & & V & & V II \\
\hline $111+$ & & + & . & + & & & & & 1111 & & & & & & I. \\
\hline I I & & & . & & 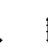 & & . & & U & & & & II & & ! \\
\hline & & I & . & II & . & . & I & & 1 & & & & . & & \\
\hline & & + &. & + & 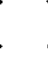 & . & . & . & I & & & & I & & I \\
\hline II IV & 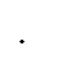 & + & I & I & . & & . & I & & & & & . & & . \\
\hline III $\mathrm{V}$ & . & . & . & I & 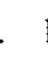 & & . & II & II & & & & . & & . \\
\hline I. & . & II & . & I & 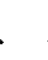 & 1 & . & . & . & & & & I & & . \\
\hline . II & II & + & . & & . & . & . & . & $\mathrm{V}$ & 4 & & & . & & . \\
\hline.+ & . & II & . & I & . & . & IV & I. & . & $\cdot$ & . & & II & & · \\
\hline I I & . & I & I & & & 1 & . & . & . & . & . & . & . & & . \\
\hline $\mathrm{I}+$ & . & . & 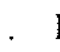 & I & 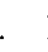 & & . & . & I & . & . & . & . & & . \\
\hline II . & . & + & $t$ & + & 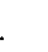 & 1 & . & II & & . & . & . & . & & . \\
\hline & & . & 1 & I & 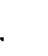 & . & & . & II & 3 & . & & . & & . \\
\hline & . & + & $t$ & + & 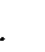 & & & . & $\mathrm{I}$ & & 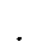 & & IV & & . \\
\hline & . & II & & & & & III & & . & . & & & IV & & V \\
\hline & II & & IV & & & & I & . & . & . & & & . & & · \\
\hline I III & . & . & & . & & . & & I & II & & & & . & & . \\
\hline III . & . & . & . & . & . & 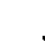 & . & I & V & 2 & & & . & & • \\
\hline . I & & + & . & + & & & . & . & $I$ & . & & & & & . \\
\hline & . & + & 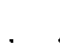 & & 1 & & I & . & . & . & & & & & . \\
\hline & . & & + & . & . & & $\cdot$ & & . & & & & I & & \\
\hline & I & & II & . & . & & . & . & . & . & . & . & . & & . \\
\hline & & . &. & + & & & & & . & & & & & & \\
\hline II IV & & . & 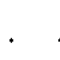 & . & & & & & I & & & & & & . \\
\hline I . & II & . & II & & & & & & . & & & & . & & \\
\hline II . & . & + & & . & & & & & . & & & & I & & . \\
\hline & & . & + & + & & & & & & & & & & & \\
\hline & & I & & II & & & IV & & & & & & & & \\
\hline & & + & & & & & & & & & & & & & \\
\hline
\end{tabular}

Ulici-Ericetum ciliaris ericetosum vagantis: 1. LOIDI (1983): tab. 35, rels.; 11-14: sub Daboecio-Ulicetum gallii ericetosum ciliaris; 2. CATALÁN (1987): tab. 9, rels. 4, 6, 7: sub Daboecio-Ulicetum gallii ericếosum ciliaris; tab. 10, rels. 3, 4: sub Daboecio-Ulicetum europaei ericetosum ciliaris; 3. BRAUN-BLANQUET (1967): tab. 25, rels. 3-9, 11, 15, 16; 4. C. NAVARro 1980, tab. 24, rels. 1-6: sub Daboecio-Ulicetum gallii ericetosum ciliaris; 5. HERRERA (1995): tab. 71, rels. 4, 5: sub Ulici europaei-Ericetum vagantis; tab. 72a, rels. 13, 15-26: sub Daboecio-Ulicetum cantabrici ulicetosum cantabrici; tab. 72b, rels. 2-7, 9: sub Daboecio-Ulicetum cantabrici genistetosum occidentalis; 6. GUINEA (1949): tab. 10, rels. 11-21, 23-26: sub Uleto-Ericetum; 7. Unpublished relevés; 8. RIVAS-MARTÍNEZ et al. (1985), tab. 2, rels. 2, 3: sub Cirsio filipenduli-Ericetum ciliaris ericetosum vagantis; 9. PERALTA DE ANDRÉs et al. (1989): tab. 2, rel. 6: sub Daboecio-Ulicetum galli; ericetosum tetralicis: 10. RiVAS-MARTÍNEZ et al. (1985): tab. 2, rels. 1, 4, 5: sub Cirsio filipenduli-Ericetum ciliaris ericetosum vagantis; 11. ONAINDIA (1986): tab. 14, rels. 7-12: sub Daboecio-Ulicetum gallii ericetosum ciliaris; 12. CÁTALÁN (1987): tab. 9, rels. 1-3, 5, 8: sub Daboecio-Ulicetum gallii ericetosum ciliaris; 13. BRAUN-BLANQueT (1967): tab. 25, rels. 1, 2, 10, 12-14; 14. C. NAVARro (1980): tab. 24, rels.7-10, sub Daboecio-Ulicetum gallii ericetosum ciliaris; 15. HERRERA (1995): tab. 72a, rel. 15: sub Daboecio-Ulicetum cantabrici ulicetosum cantabrici; 16. PERALTA DE ANDRÉs et al. (1989): tab. 2, rel. 5: sub Daboecio-Ulicetum gallii:Ulici (gallii) humilis-Ericetum vagantis typicum: 17. FERNÁNDEZ PRIETO \& LOIDI (1984b): tab. 11, rels. 1-8; ulicetosum maritimi: 18. FERNÁNDEZ PRIETO \& LOIDI (1984b): tab. 11, rels. 9-12.; Genisto occidentalis-Ulicetum maritimi: 19. FERNÁNDEZ PRIETO \& LOIDI (1984b): tab. 12, 6 rels. 20. C. NAVARRO (1980): tab. 26, 5 rels. 
Table 2. Daboecienion cantabricae (excl. Ulici-Ericetum ciliaris). 1-7 Vaccinio myrtilli-Ulicetum gallii; $8-13$ Erico tetralicis. Ulicetum gallii (8-12 ulicetosum gallii. 13 juniperetosum alpinae): 14-29 Ulici europaei-Ericetum vagantis (14-24 ericetosum vagantis, 25-29 genistetosum occidentalis); $30-34$ Halimio umbellati-Daboecietum cantabricae ( 30 daboecietosum cantabricae, 31 ulicetosum gallii, 32 genistetosum pilosae, 33 genistelletosum tridentatae, 34 ericetosum umbellatae); $35-38$ Arctostaphylo crassifoliae-Daboecietum cantabricae (35 daboecietosum cantabricae. 36 ulicetosum gallii, 37-38 ulicetosum europaei); 39-41 Ericetum scopario-vagantis (39-40 ericetosum scopariae, 41 lavanduletosum pedunculatae); 42 Daboecio cantabricae-Ericetum aragonensis.

\title{
Differentials of the associations and subassociations
}

Vaccinium myrtillus

Erica tetralix

Carex binervis

Nardus stricta

Juniperus alpina

Rubus ulmifolius

Quercus robur

Ulex europaeus

Genista occidentalis

Smilax aspera

Halimium umbellatum

Halimium alyssoides

Genista tridentata

Erica umbellata

Arctostaphylos crassifolia

Thymelaea ruizii

Erica scoparia

Lavandula pedunculata

Erica aragonensis

Agrostis delicatula

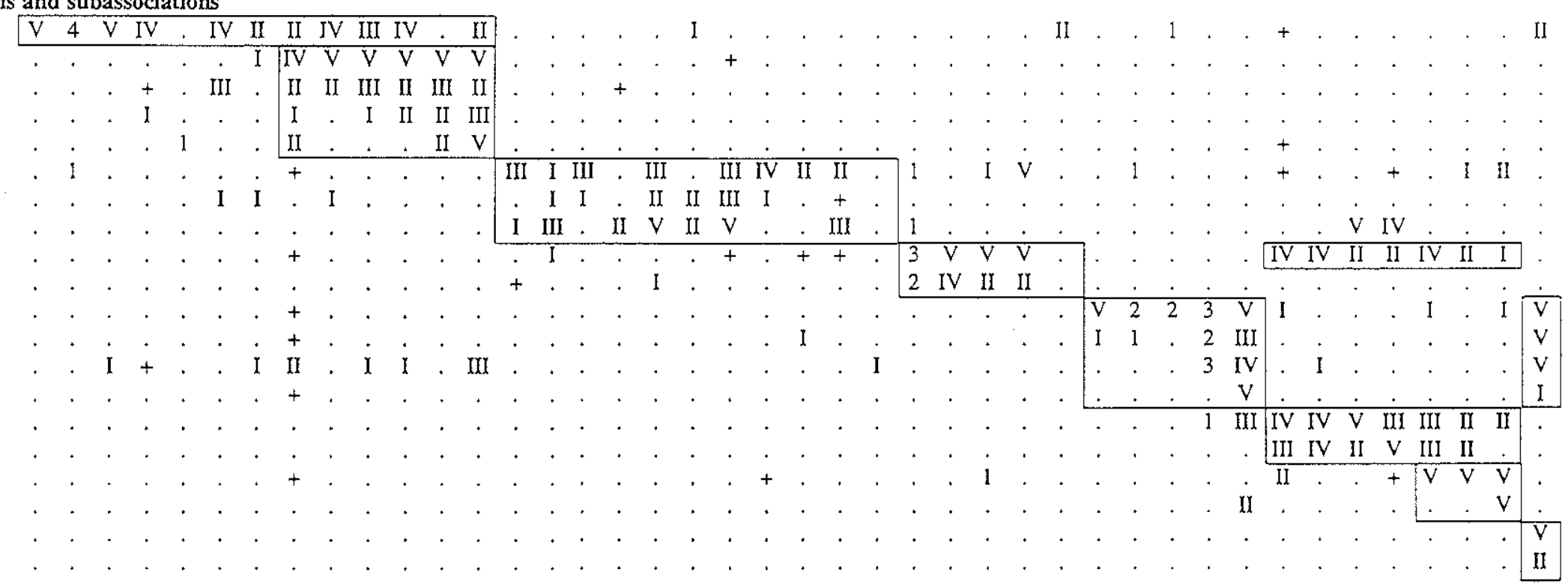

Character species of the high-rank units

Erica vagans

Calluna vulgaris

Erica cinerea

Daboecia cantabrica

Potentilla erecta

Agrostis curtisii

Ulex gallii

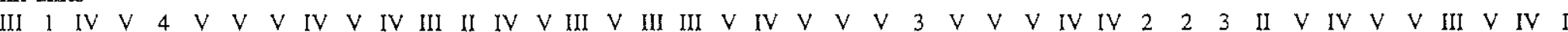

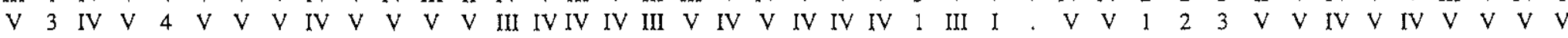

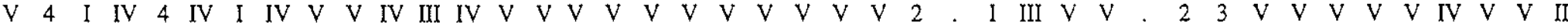

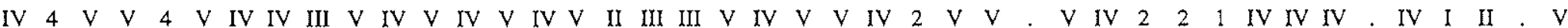

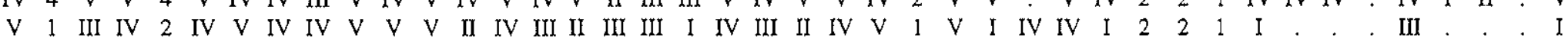
Avenula sulcato

IV 3 . III $2 \mathrm{~V}$ II $\mathrm{V}$ IV $\mathrm{V}$ IV $\mathrm{V} V$ II II III $\mathrm{V}$. III II III IV II IV

$\checkmark$ III 1 l 2 I III II III. Serratula tinctoria

III 2 IV IV 4 III IV V III V II $\mathrm{V} V \mathrm{~V} V \mathrm{~V} V \cdot \mathrm{V}+\mathrm{V} V \mathrm{~V} V 2 \mathrm{~V}$ IV V V .2 IV $\cdot \mathrm{I} \cdot \mathrm{i} \cdot \mathrm{in}$

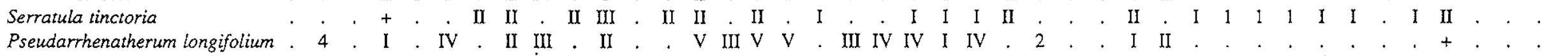

Arenaria montana

+ V II . II . II I . II

Carex pilulifera

III II II . . III . I I . . $++\cdot+$

\begin{abstract}
I III
\end{abstract}
1 III I 
Cirsium filipendulum Polygala serpyllifolia Cistus salviifolius

Genista pilosa

Simethis mattiazzi

Euphorbia polygalifolia

Lithodora prostrata

Lithodora diffusa

Euphorbia angulata

Laserpitium dufourianum

Viola lactea

Genista anglica

Genista micrantha

Tuberaria globulariifolia

Erica ciliaris

\section{Companions}

Pteridium aquilinum

Brachypodium rupestre

Stachys officinalis

Danthonia decumbens

Potentilla montana

Agrostis capillaris

Festuca rubra agg.

Deschampsia flexuosa

Hypericum pulchrum

Teucrium scorodonia

Pimpinella saxifraga

Polygala vulgaris

Viola silvestris agg.

Anthoxanthum odoratum

Hypochoeris radicata

Lotus corniculatus

Quercus pyrenaica

Cruciata glabra

Galium saxatile

Blechnum spicant

Molinia caerulea s.l.

Carex flacca

Lathyrus linifolius

Sanguisorba minor

Crataegus monogyna

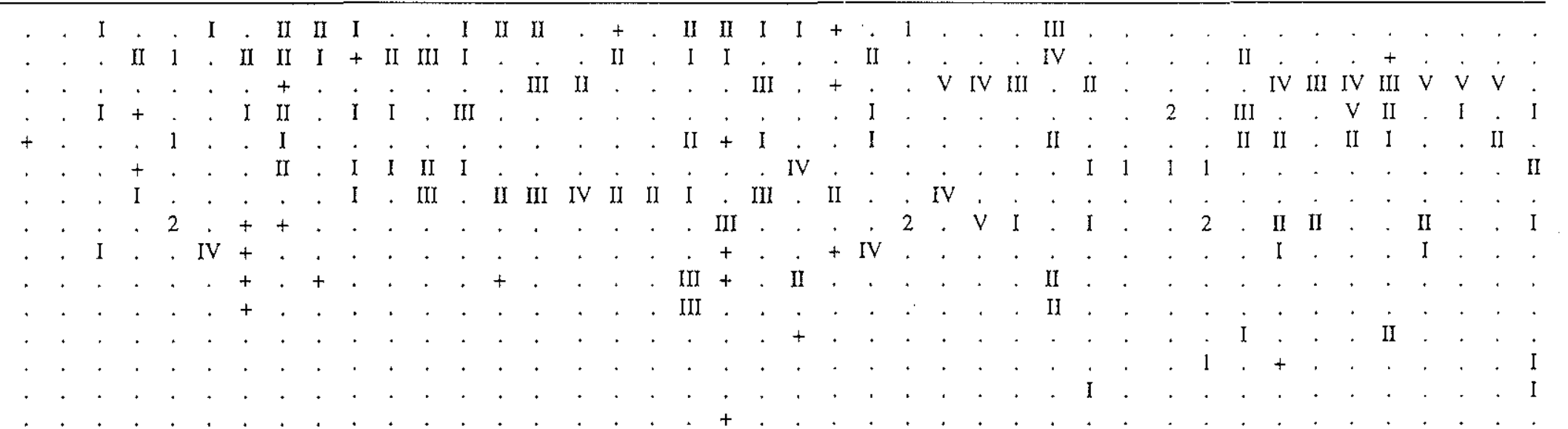

III 3 III III. IV V II II III II .

$\mathrm{V}$ III $\mathrm{V}$ V IV IV $\mathrm{V}$ III II IV $\mathrm{V} 1$. . IV $\mathrm{V}$

III III I III II

. 2 I II . . IV II III.+ . I IV III II IV IV. IV IV III III $V$

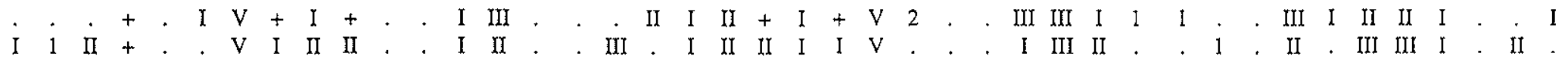

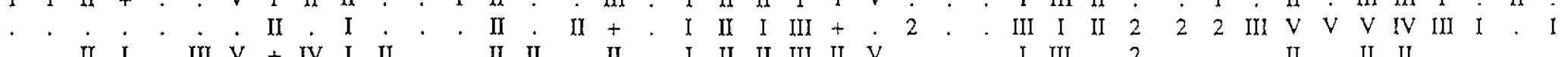

. II $\mathrm{I}$ III $\mathrm{V}+\mathrm{IV}$ I II . . II II . II . I II II III II $\mathrm{V}$. . I III .

2 II I . IV $+\mathrm{V}+\mathrm{I}$. . II . . III . I II + . I III I. . . III . . . III I . II . II

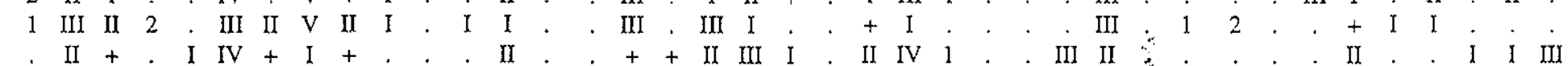

I II I III + I . III. IV II II II II I I IV IV 2 II . II

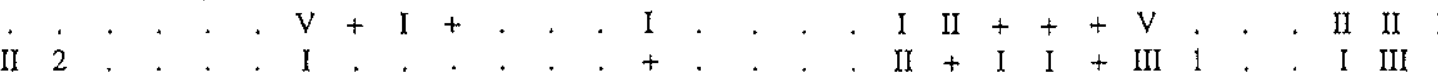

II

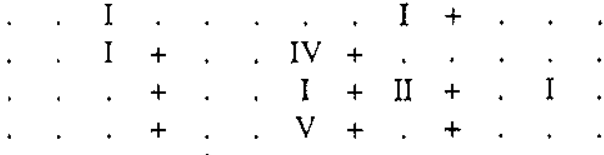

II + II I III II . II II 1 II

$\begin{array}{ccccccccccc}\text { I } & \text { III } & \text { II } & . & \text { II } & \text { II } & 1 & \text { II } & . & \text { III } & \text { II } \\ \text { I } & \text { I } & \cdot & . & . & . & . & . & . & . & \text { I } \\ \text { II } & \text { II } & + & . & + & \text { II } & . & . & . & . & \text { II }\end{array}$

IV 2 II III $\cdot$ IV $+\dot{\text { III }}+\dot{ }+\dot{\text { IV }}$ I

IV 2 II III IV + V . IV I . I . II . I I II 1 IV . . . III

\begin{tabular}{cccccccccc}
. & II & I &. &. & II & 2 &. &. & III \\
II & + & II & + & I &. &. &. &. &. \\
. & I & II & I & IV &. &. &. & I \\
\hline
\end{tabular}

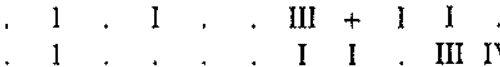

iI + II II

I +

$+1$

II

. . . . I I . . . I . . . . I II I +. . . . II . .

I $\mathrm{I}+.+$ II $\cdot$ II . III II

I +

+++ II $i$ II . IV III II 
Asphodelus albus

Hieracium pilosella

Helianthemum nummularium

Seseli cantabricum

Solidago virgaurea

Prunus spinosa

Cytisus scoparius

Briza media

Erica arborea

Scilla verna

Jasione montana

Prunella vulgaris

Teucrium pyrenaicum

Hedera helix

Jasione laevis

Gentiana pneumonanthe

Thymus britannicus

Centaurea nigra s.l.

Holcus lanatus

Rubia peregrina

Quercus faginea

Dorycnium pentaphyllum

Anemone nemorosa

Crocus nudiflorus

Achillea millefolium

Juniperus communis

Cuscuta epithymum

Pedicularis sylvatica

Trifolium pratense

Cytisus cantabricus

Luzula campestris

Rubus sp.

Thymus pulegioides

Pulmonaria longifolia

Salix atrocinerea

Andryalo integrifolia

Galium verum

Euphorbia dulcis

Erica lusitanica

Lonicera periclymenum

Arbusus unedo

Buxus sempervirens

. II +

$\begin{array}{ll}\text { II } & + \\ \text { II }\end{array}$

I..

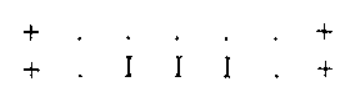

III + .

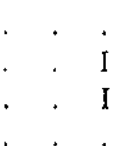

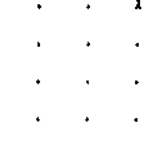

$+$

II $:$ i.++

1 . I II + II II

II.$++. \cdot . \cdot 1$

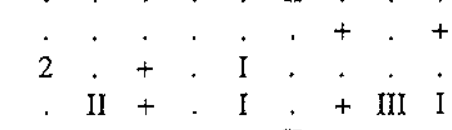

. II

II

iv +

ill 1

I.

II +

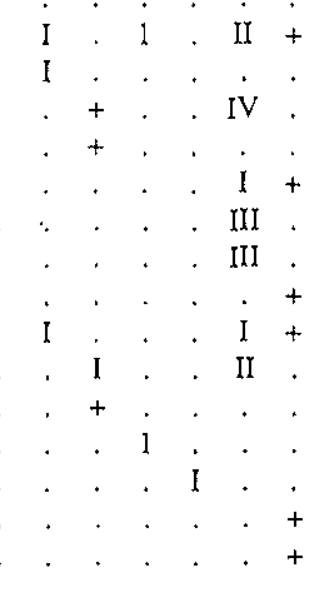

III .

II. IV

IV .

III III II .

III

. IV I II

iI $\dot{I}+$

III .

$\cdot \cdot+\cdot+\cdot \dot{j}+\dot{1}$

i ++++ III

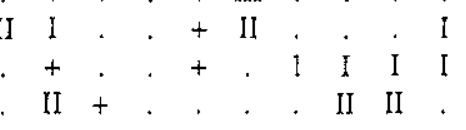

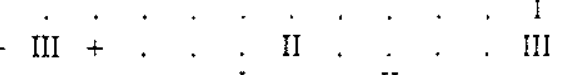

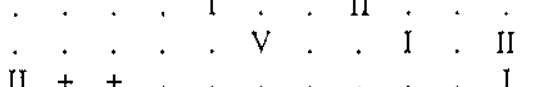

$\mathrm{II}++. . \quad \cdot+\frac{.}{\mathrm{V}} \dot{\mathrm{V}} \mathrm{I}$

II III

$$
\text { i } . \quad . \quad \text { II }
$$

II . $\cdot$ II $\cdot$ III

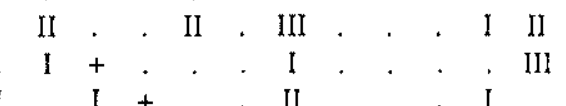

$+\cdot \cdot \cdot \cdot \cdot \cdot \cdot \cdot$

III . . + III
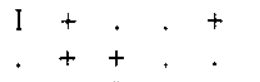

$++$

II $11 \cdot \cdot \cdot 1$

III 1 
Quercus rotundifolia

Viola canina

Ranunculus bulbosus s.l.

Amelanchier ovalis

Sorbus aucuparia

Enthronium dens-canis

Leontodon taraxacoides

Linum catharticum

Hepatica nobilis

Euphorbia amygdaloides

Plantago lanceolata

Veronica officinalis

Bromus erectus

Carlina vulgaris $\mathrm{s} .1$.

Melampyrum pratense

Picris hieracioides

Origanum vulgare

Rosa pimpinellifolia

Eryngium campestre

Filipendula vulgaris

Lavandula Latifolia

Genista scorpius

Carex caryophyllea

Cytisus commutatus

Fagus sylvatica

Rosa pendulina

llex aquifolium

Huperzia selago

Festuca ovina agg.

Leucanthemum vulgare $s .1$

Leontodon hispidus s.l.

Allium ericetorum

Veronica chamaedrys

Senecio adonidifolius

Prunella hastifolia

Globularia nudicaulis

Scirpus germanicus

Scorzonera humilis

Hypericum humifusum

Silene nutans

Physospermum cornubiense

Sorbus aria

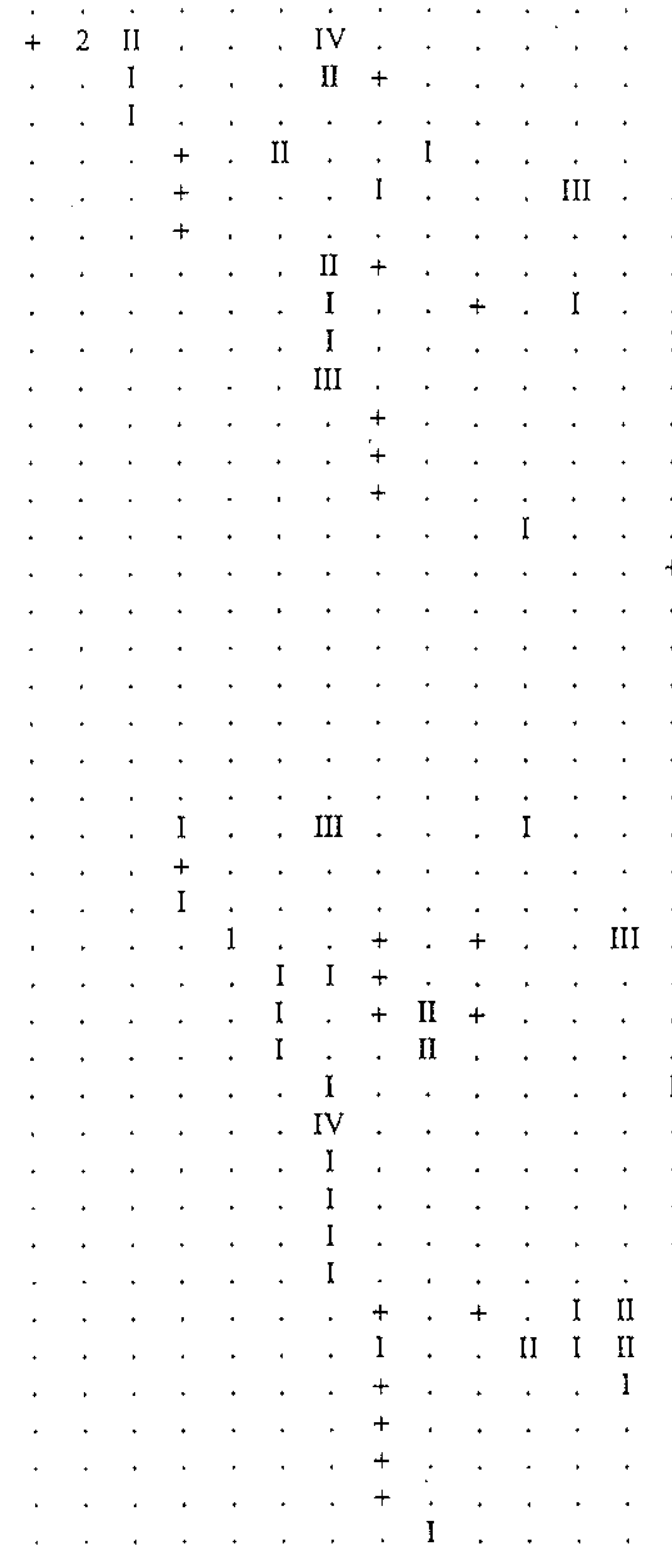

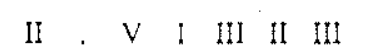

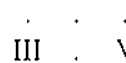

1

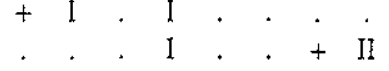

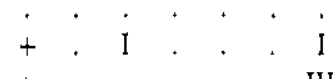

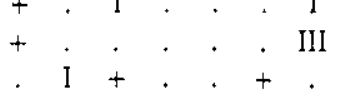

III

II

II.

$+$

I

11

$++$.

$1+I V$

I II II I

+ IV II II

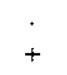




\section{Frangula alnus}

Rhamnus alaternus

Xolantha tuberaria

Serratula seoane

Centaurea debeaux

Dactylis glomerata

Tuberaria guttata

Thymus praecox

Trifolium montanum

Satureja vulgaris

Carlina corymbosa

Trifolium ochroleucon

Carex humilis

Spiraea obovata

Geum sylvaticum

Helichrysum stoechas

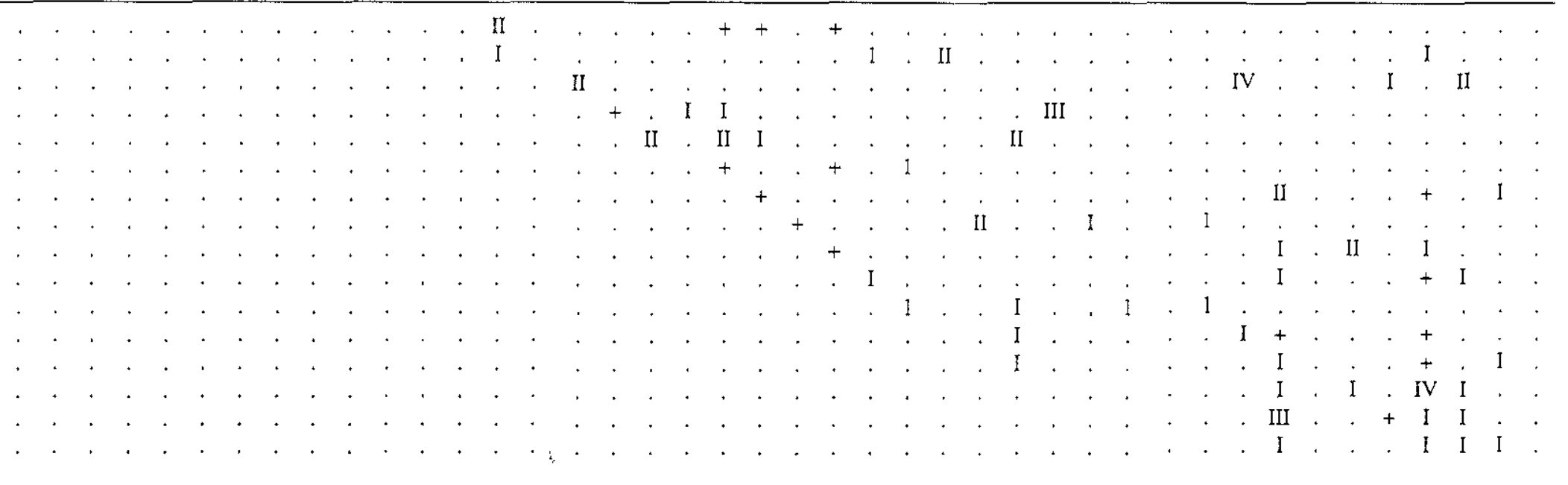

Vaccinio myrtilli-Ulicetum gallii: 1. LOMI (1983): tab. 35, rels. 1-10: sub Daboecio-Ulicetum gallii vaccinietosum myrtilli; 2. ONAINDIA (1986): tab. 14, rel. 2: sub Daboecio-Ulicetum gallii ericetosum ciliaris; tab. 15, 3 rels.: sub Daboecio-Ulicetum gallii vaccinietosum myrtilli; 3. RIVAS-MARTíneZ et al. (1991): rel. 2: sub Daboecio-Ulicetum cantabrici genistetosum pilosae: rels. 3-6: sub Daboecio-Ulicetum cantabrici vaccinietosum myrtilli; 4. Unpublished relevés; 5. TARAZONA \& ZALDfVAR (1987): tab. 1, rels. I-4: sub Daboecio-Ulicetum gallii; 6. CATALÁN (1987): tab. 7, rels. 2. 3: sub Daboecio-Ulicetum gallii ulicetosum gallit; tab. 7, rels. 15, 18, 19, 21: sub Daboecio-Ulicetum gallii vaccinietosum myrtilli, tab. 8, rel. 3: sub com. Calluna vulgaris y Vaccinium myrtillus: 7. VAN DEN BERGHEN (1973): pag. 94, col. 1, 14 rels.: sub Pteridio-Ericetum vagantis, Erico tetralicis-Ulicetum gallii typicum: 8 . Unpublished relevés: 9 . CATALÁ (1987): tab. 7, rels. 7, 16, 17. 20,22, 23: sub Daboecio-Ulicetum gallii vaccinietosum myrtilli, tab. 8, rels. 1, 2: sub com. Calluna vulgaris y Vaccinium myrtillus; 10. HERRERA (1995): tab. 73, rels. 1-17: sub Erico tetralicis-Ulicetum gallii ulicetosum gallii; tab. 74, 4 rels.: sub com. Calluna vulgaris y Vaccinium myrtillus. 11. Unpublished relevés; 12. TARAZONA \& ZALDfVAR (1987): tab. 1, rels. 5-10: sub Daboecio-Ulicetum gallii ericetosum tetralicis; juniperetosum alpinae: 13. HERRERA (1995): tab. 73, rels. 18-25. Ulici-Ericetum vagantis typicum: 14. HERRERA (1995): tab. 71, rels. 1, 3; tab. 72a, rels. 1-12: sub Daboecio-Ulicetum cantabrici; 15. LomI (1983): tab. 34, 9 rels.: sub Daboecio-Ulicetum gallii ulicetosum gallii; 16. ONAINDIA (1986): tab. 14, rels. 1, 3-6: sub Daboecio-Ulicetum gallii ulicetosum gallii; tab. 16,3 rels.: st·b Daboecio-Ulicetum gallit tuberarietosum; 17. CATALÁN(1987); tab. 7, rels. 1,4-6,8-14: sub Daboecio-Ulicetum gallii ulicetosum gallii, tab. 10, rels. 1-2: sub Daboecio-Ulicetum europaei ulicetosum europaei; 18. GUINEA (1949): tab. 10, rels. 1-10, 22, 27-40: sub Uleto-Ericetum; 19. BASCONES (1978): tab. 16, rels. 7. 8, 10. 11, 14: sub Daboecio-Ulicelum europaei ulicetosum gallii: 20. BRAUN-BLANQUET (1967): tab. 26, 20 rels.: sub Daboecio-Ulicetum europaet lithodoretosum; tab. 27, 8 rels.: sub Daboecio-Ulicetum europaei ulicetosum gallii: 21. LoDI et al. (1992): tab. 5, rels. 1-21: sub Daboecio-Ulicetum gallii ulicetosum gallii; 22. FERNÁNDEZ PRIETO \& LOIDI (1984a): tab. 1, rels. 1-11: sub Daboecio-Ulicetum gallii; 23. Unpublished relevés; 24. vaN DEN BERGHEN (1973): pag. 94, col. 2, 20 rels.: sub Pteridio-Ericetum vagantis; genistetosum occidentalis: 25. HeRRERA (1995): tab. 71, rel. 2: sub Ulici-Ericetum vagantis; tab. 72b, rels. 1, 8: sub Daboecio-Ulicetum cantabrici genistetosum occidentalis; 26. C. NAVARRO (1980): tab. 25. 10 rels.: sub Daboecio-Ulicetum gallii genistetosum occidentalis; 27. ONANDIA (1986): tab. 17, 8 rels.: sub Daboecio-Ulicetum gallii genistetosum occidentalis; 28. LoID et al. (1992): tab. 5, rels. 26-32: sub Daboecio-Ulicetum gallii genistetosum occidentalis; 29. BÁscones (1978): tab. 16, rels. 1-6, 9, 12, 13: sub Daboecio-Ulicetum europaei ulicetosum gallii. Halimio umbellati-Daboecietum cantabricae typicum: 30. FERNÁNDEZ PRIETO \& LOID1 (1984a): tab. 2, rels. 1-6: ulicetosum gallii: 31. FERNÁNDEZ PRIETO \& LODI (1984): tab. 1, rels. 12, 13: sub Daboecio-Ulicetum gallii halimietosum umbellati; genistetosum pilosae: 32. FERNÁNDEZ PRIETO \& LODDI (1984a): tab. 2, rels. 7. 8; genistelletosum tridentatae: 33. FERNÁNDEZ PRIETO \& LODI (1984a): tab. 2, rels. 9-11; ericetosum umbellati: 34. LOTDI \& FERNÁNDEZ PRIETO (1986): tab. 7, 7 rels. Arctostaphylo crassifoliae-Daboecietum cantabricae typicum: 35. GARCíA-MIJANGOS (1994): tab. 36, rels. 1-24, 26-28: sub Halimio umbellati-Daboecietum cantabricae arctostaphyletosum crassifoliae; ulicetosum gallii: 36. TARAZONA \& ZALDIVAR (1987): tab. 2, 5 rels.: sub Daboecio cantabricae-Ulicetum gallii arctostaphyletosum crassifoliae; ulicetosum europaei: 37. PERALTA DE ANDRÉS (1992): tab. 25. 8 rels.: sub Ulici-Ericetum vagantis; 38. Unpublished relevés. Ericetum scopario-vagantis typicum: 39. GARCiA-MUANGOS (1994): tab. 36, rel. 25.; sub Halimio umbellati-Daboecietum cantabricae arclostaphyletosum crassifoliae, tab. 37, rels. 1-15; 40. Unpublished relevés; lavanduletosum pedunculatae: 41. GARCiA-MIJANGOS (1994): tab. 37, rels. 16-20. Daboecio cantabricae-Ericetum aragonensis: 42. FERNÁNDEZ PRIETO \& LOIDI (1984a): tab. 3, 6 rels. 


\section{Table 3}

Genistion micrantho-anglicae. 1-11 Genisto anglicae-Ericetum vagantis (1-7 ericetostum vagantis, 8 cistetosum laurifolii, 9 ericetosum tetralicis, 10 ericetosum scopariae, 11 arctostaphyletosum crassifoliae); $12-15$ Genisto anglicae-Daboecietum cantabricae (12-14 daboecietosum cantabricae, 15 ulicetosum gallii); 16-17 Euphorbio polygalifoliae-Ericetum tetralicis (16 ericetosum tetralicis, 17 ericetosum vagantis).

No. of column

No. of relevés

$\begin{array}{lllllllllllllllll}8 & 19 & 5 & 1 & 5 & 7 & 6 & 5 & 7 & 7 & 25 & 1 & 10 & 15 & 6 & 1 & 7\end{array}$

Differentials of the Genisto anglicae-Daboecietum cantabricae

Daboecia cantabrica

Cistus salviifolius

$\left[\begin{array}{llll}\mathrm{I} & \mathrm{V} & \mathrm{V} & \mathrm{V} \\ \mathrm{I} & \mathrm{II} & \mathrm{I} & \mathrm{I}\end{array}\right] \cdot \mathrm{I}$

Differentials of the Euphorbio polygalifoliae-Ericetum tetralicis

Euphorbia polygalifolia

Gentiana pneumonanthe

Succisa pratensis

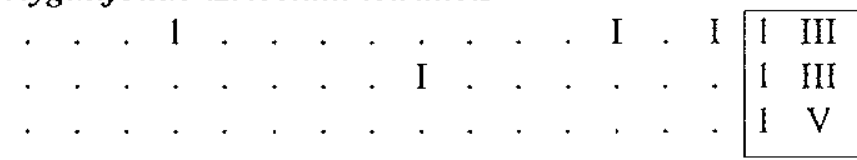

Differentials of the subassociations

Halimium viscosum

Trifolium ochroleucon

Daphne cneorum

Erica aragonensis

Sanguisorba officinalis

Cistus laurifolius

Erica tetralix

Erica scoparia

Arctostaphylos crassifolia

Lavandula pedunculata

Vaccinium myrtillus

Halimium alyssoides

Erica umbellata

Ulex gallii

Laserpitium dufourianum

Molinia caerulea s.1.

$$
\begin{aligned}
& \cdot+\cdot \cdot \text { III } \cdot \text { IV } \cdot \text { IV } \\
& \text { I V } \\
& \text {. III } \\
& \text {. III } \\
& \text { I. . . . V . IV } \\
& \text {. I . . . . V II . . . } 1 \text { V } \\
& \text {. . . . . . III V . . . . . } \\
& \text {. . . . . . . IV . I I . . } \\
& \text {. +. . . . II . II }
\end{aligned}
$$

Character species of the high-rank units and companions

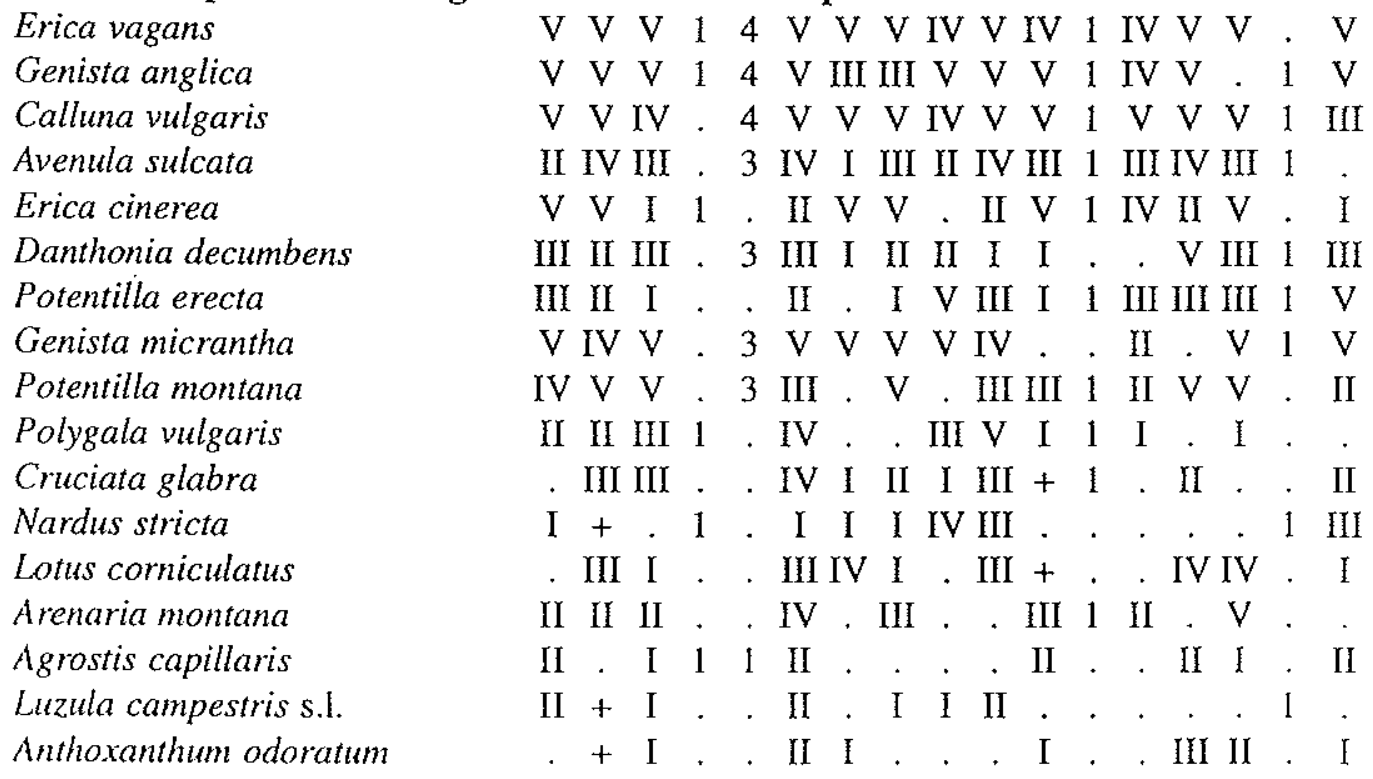


No. of column 1233456678991011121314151617

Brachypodium rupestre. Agrostis curtisii

Festuca rubra agg.

Stachys officinalis

Quercus pyrenaica

Genista pilosa

Hypericum pulchrum

Hieracium pilosella s.1.

Genista occidentalis

Pteridium aquilinum

Briza media

Simethis mattiazzi

Carex pilulifera

Halimium umbellatum

Juncus squarrosus

Carex flacca

Galium verum

Juniperus communis

Galium pinetorum

Holcus lanatus

Lathyrus linifolius

Juncus acutiflorus

Achillea millefolium

Serratula tinctoria

Lotus pedunculatus

Juncus effusus

Filipendula vulgaris

Erica arborea

Carum verticillatum

Thymelaea ruizii

Deschampsia flexuosa
12545161801011121314151617

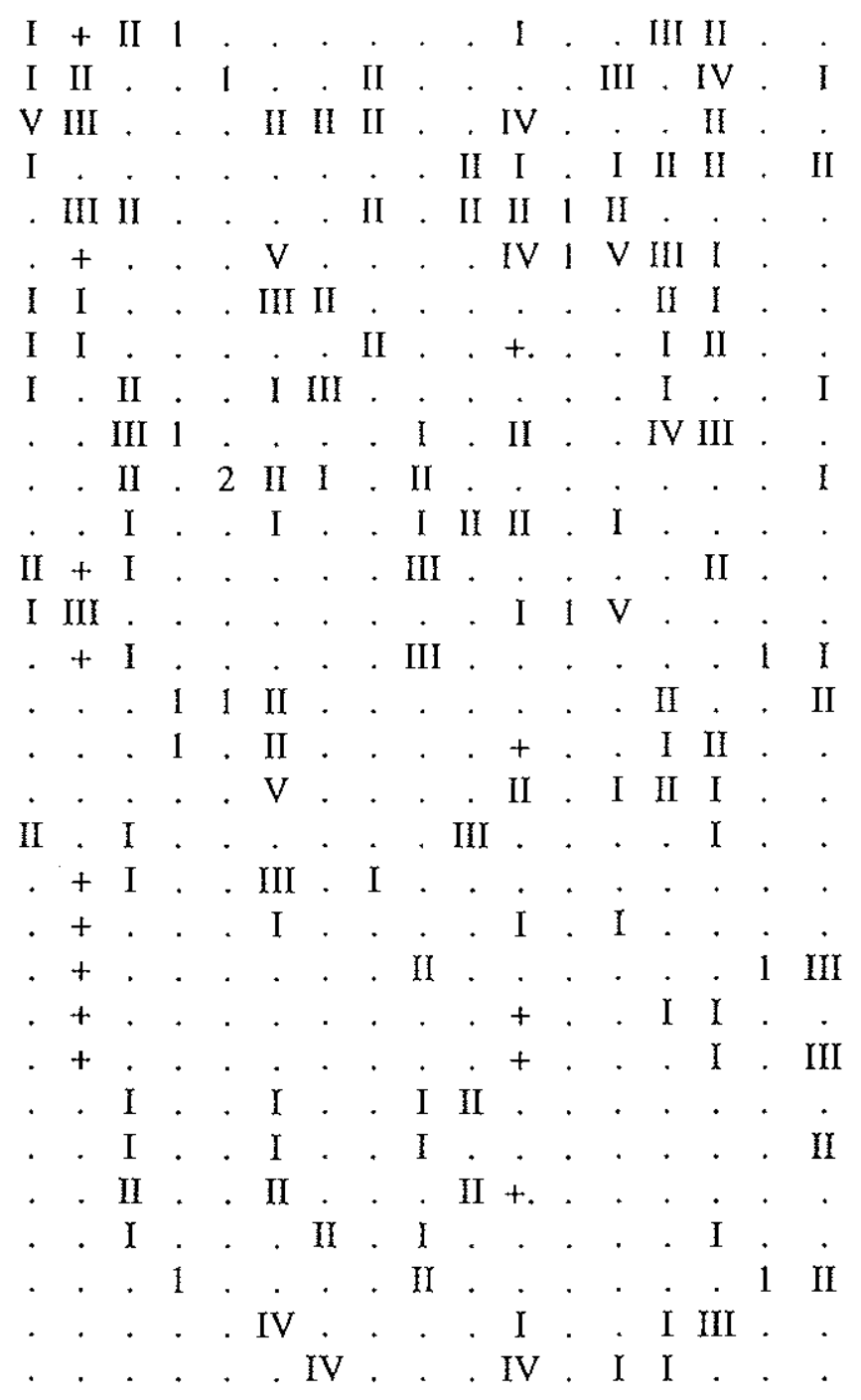

Genisto anglicae-Ericetum vagantis typicum: 1. RIVAS-MARTINEZ (1979): tab. 19, 8 rels. 2. TARAZONA \& ZALDíVAR (1987): tab. 6, rels. 1-19. 3. G. NAVARRO (1986): tab. 43, rels. 1-5. 4. Unpublished relevé. 5. FERNÁNDEZ PRIETO \& LOIDI (1984a): tab. 4, 4 rels. 6. MEDRANO MORENO (1994): tab. 34, 7 rels. 7. MENDiOLA (1983): 258, 6 rels.; cistetosum laurifolii: 8. TARAZONA \& ZALDÍVAR (1987): tab. 6, rels. 20-24; ericetosum tetralicis: 9. G. NAVARRO (1986): tab. 51, 7 rels.: sub Genisto anglicae-Ericetum tetralicis; ericetosum scopariae: 10. G. NAVARRO (1986): tab. 43, rels. 6-12; arctostaphyletosum crassifoliae: 11. PERALTA DE ANDRÉS (1992): tab. 23, 25 rels. Genisto anglicae-Daboecietum cantabricae typicum: 12. TARAZONA (1984): tab. 4, rels. 22: sub Genisto anglicae-Ericetum vagantis daboecietosum cantabricae. 13. TARAZONA \& ZALDÍVAR (1987): tab. 3, 10 rels.: sub Genisto anglicae-Ericetum vagantis daboecietosum cantabricae. 14. PERALTA DE ANDRÉS (1992): tab. 24, 15 rels.: sub Genisto pilosae-Daboecietum cantabricae; ulicetosum gallii: 15. Unpublished relevé. Euphorbio polygalifoliae-Ericetum tetralicis ericetosum tetralicis: 16. FERNÁNDEZ PRIETO \& LOIDI (1984a): tab. 5, rel. 1.; ericetosum vagantis: 17. FERNÁNDEZ PRIETO \& LOIDI (1984a): tab. 5, rels. 2-8. 


\section{Table 4}

Ericion umbellatae. 1-19 Genisto pilosae-Ericetum aragonensis (1-8 ericetosum aragonensis, 9-10 cistetosum laurifolii, 11-12 genistetosum anglicae, 13-16 cytisetosum oromediterranei, 17-18 daboecietosum cantabricae, 19 pterospartetosum tridentatae); 20-24 Calluno vulgaris-Genistetum occidentalis (20 genistetosum occidentalis, 21-22 ericetosum aragonensis, 23 genistetosum micranthae, 24 lavanduletostm pedunculatae).

No. of column

No. of relevés

$\begin{array}{llllllllllllllllllllllll}7 & 3 & 7 & 6 & 3 & 6 & 1 & 5 & 4 & 2 & 2 & 4 & 2 & 2 & 3 & 3 & 3 & 2 & 6 & 6 & 4 & 1 & 2 & 6\end{array}$

Differential species of the asssociations and subassociations

Genista pilosa

Vaccinium myrtillus

Luzula lactea

Halimium umbellatum

Deschampsia iberica

Agrostis curtisii

Cistus laurifolius

Genista anglica

Cytisus purgans

Daboecia cantabrica

Genista tridentata

Simethis mattiazzi

Arctostaphylos crassifolia

Genista occidentalis

Deschampsia flexuosa

Melampyrum pratense

Brachypodium rupestre

Lathyrus linifolius

Festuca paniculata s.1.

Erica scoparia

Genista micrantha

Genista tinctoria

Halimium viscosum

Cistus albidus

Genista scorpius

Lavandula pedunculata

Avenula mirandana

Geum sylvaticum

Thymus mastichina

Thymus vulgaris

Cephalanthera longifolia

\begin{tabular}{|c|c|c|c|c|c|c|c|c|c|c|c|c|c|c|c|c|c|c|c|c|}
\hline & & & & & & & & & & & & & & & & & & & & \\
\hline & 3 & II & I & $3 \mathrm{IV}$ & & IV & & 2 & 1 & 4 & 1 & & 2 & & & · III & $\mathrm{I} \mid$. & & & \\
\hline II & & II I & & $1 \mathrm{IV}$ & 1 & & & . & . & . & 1 & 1 & 3 & 1 & & . I & . & . & 1 & \\
\hline 11 & 3 & IV I & & . III & & . & & 2 & 1 & 3 & & & 1 & & 1 & . V &. & & & \\
\hline 11 & 3 & & & . III & & & & 2 & 2 & 1 & 1 & & & & 2 & $2 \mathrm{III}$ & & & & \\
\hline 11 & & I & V & . IV & & . & & & & & 2 & 1 & 2 & & & $2 I$ & . & & & \\
\hline H & 2 & III & & . . & & . & 1 & 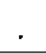 & 2 & 1 & & 1 & & & & . III & & & & \\
\hline & & & & 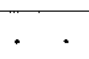 & & . & 4 & 2 & & & ${ }^{\circ}$ & & & & &.$\quad$ & IV & 1 & & \\
\hline & & III & & . & & . & . & . & 2 & 4 & . & $\dot{0}$ & . & & & . & . & & & \\
\hline & & . & . & . I & . & II & 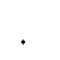 & . & . &. & 2 & 2 & 3 & 3 & & & I & & & \\
\hline & & . & . & . & & . & . & . & . & . & . & . & & & 3 & $2]$. & . & & & \\
\hline l & & . & . & . & 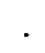 & . & . & . & . & . & . & . & . & & & $\cdot \mathrm{V}$ & $\pi$ & & & \\
\hline & 1 & . & . & . & & . & & . & . & & . & . & . & & & . III & I & . & & \\
\hline & & . & & 1 & & & 1 & & . & & . & & & & & I & V & 4 & 2 & $2 \mathrm{III}$ \\
\hline & & . & & 2 & & & & & & & . & & 2 & & & & V & 4 & & $2 \mathrm{~V}$ \\
\hline & & . & & . & & IV & & . & . & & . & & & 2 & & & V & 4 & & \\
\hline & . & I & . & . & & . & 1 & . & . & $\cdot$ & . & & & & & & III & 1 & & II \\
\hline & & . & . & . & 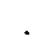 & . & . & . & . & . & . & 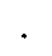 & & & . & & I & 1 & & 2 \\
\hline & & . & . & . & & . & . & . & . & . & . & & & & 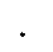 & & I & 1 & & $1 \mathrm{I}$ \\
\hline & & & & . & & . & . & & . & & . & & & & & . & III & & & $1 \mathrm{IV}$ \\
\hline & & & 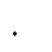 & . & & & . & & . & 1 & 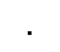 & & & & & & 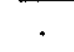 & & & 2 \\
\hline & & & . & . & & & . & . & . & & ${ }^{\circ}$ & & & & & . & & & & 2 \\
\hline & & & 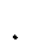 & & & & & & 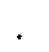 & & & & & & & & & & & $V$ \\
\hline & & & & ${ }^{\circ}$ & & . & 1 & & 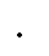 & & $\cdot$ & & & & & & $I$ & & & \\
\hline & & . & . & . & & . & . & & 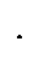 & • & . & & & & . & . & I & 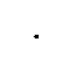 & & . \\
\hline & & & & . & & & & & & . & . & & & & & & . & . & & 2 \\
\hline & & & & . & & & 2 & & & 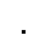 & . & & & & & & & & & \\
\hline & & & . & & & & $\cdot$ & & 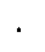 & & ${ }^{\circ}$ & & & & & & & & & \\
\hline & & & & & & & & & & & & & & & 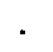 & & . & & & \\
\hline & & & & . & & & $\cdot$ & & 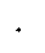 & & & & & & & & & & & $\cdot 1$ \\
\hline & & & & . & & & $\cdot$ & & & & & & & & & & $\cdot$ & & & 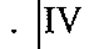 \\
\hline & & & & & & & & & & & & & & & & & & & & \\
\hline
\end{tabular}

Character species of the high-rank units

Calluna vulgaris

Arenaria montana

Avenula sulcata

Erica cinerea

Halimium alyssoides

Erica vagans

Halimium ocymoides

Xolantha tuberaria

Cistus salviifolius

Potentilla erecta

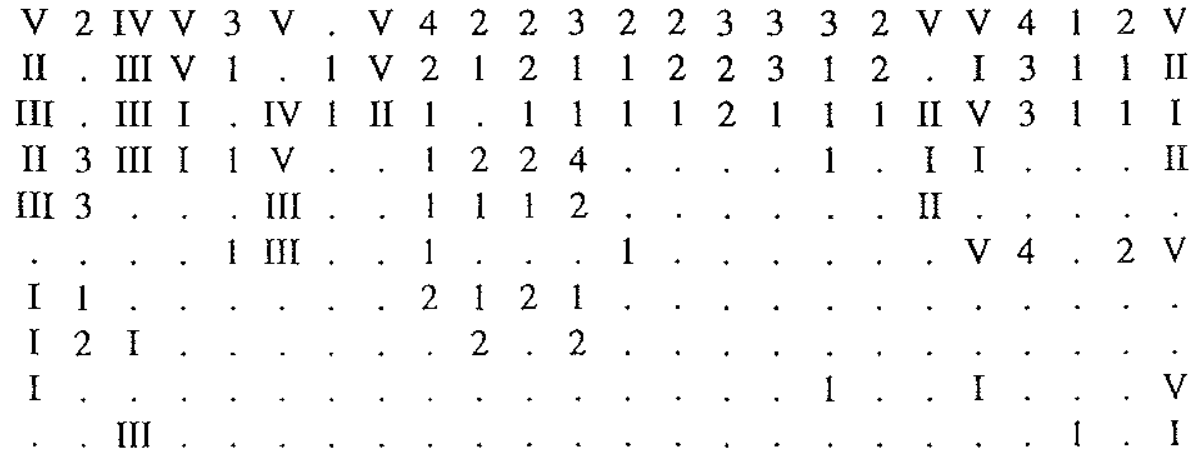


No. of column

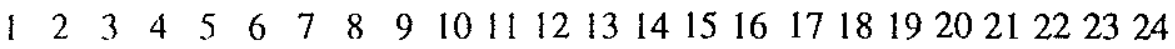

Companions

Erica aragonensis

Cytisus scoparius

Pinus syluestris

Lotus corniculatus

Hypericum pulchrum

Anthoxanthum odoratum

Viola silvestris agg.

Erica arborea

Teucrium scorodonia

Quercus pyrenaica

Pteridium aquilinum

Genista polygaliphylla

Potentilla montana

Agrostis castellana

Hypochoeris radicata

Danthonia decumbens

Cruciata glabra

Juniperus communis

Viola montcaunica

Jasione carpetana

Hieracium pilosella s.l.

Nardus stricta

Sedum brevifolium

Digitalis parviflora

Antennaria dioica

Agrostis delicatula

Veronica officinalis

Jasione laevis

Hieracium murorum

Stachys officinalis

Asphodelus aestivus

Luzula forsteri

$$
\begin{aligned}
& \begin{array}{llllllllllllllllllllllll}
\mathrm{V} & 3 & \mathrm{~V} & \mathrm{~V} & 3 & \mathrm{~V} & 1 & \mathrm{~V} & 4 & 2 & 2 & 4 & 2 & 2 & 3 & 3 & 3 & 2 & \mathrm{~V} & . & 4 & 1 & \text {. }
\end{array} \\
& \text { II I III II } 1 \text { I }
\end{aligned}
$$

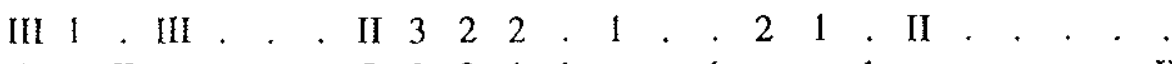

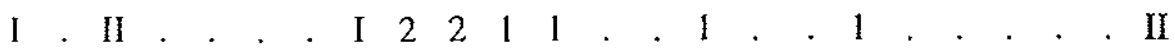

$$
\begin{aligned}
& \text { II . . II . II . III } 1 \text { l } 1 \text {. . . . . . } 1 \text {. } \\
& \text { II . . Il } . \quad . \quad \text { IY } 1 \text { I }
\end{aligned}
$$

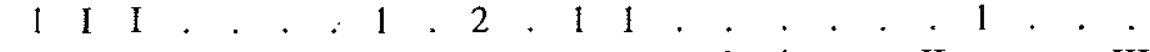

$$
\begin{aligned}
& \text {. . V I . } 1 \text { IV . . . . . . . } 21 \text {. } 2 \text { II }: \text {. III }
\end{aligned}
$$

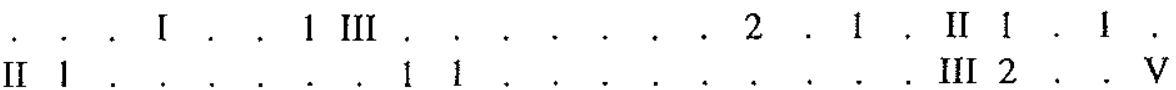

$$
\begin{aligned}
& \text { I. I I : . . . . } 1 \text {. . . . . . . } 1 \text { I . . . . I } \\
& \text {. . . } 2 \text {. } 1 \text {. . . . . . . . } 1 \text {. } 1 \text {. II } 2 \text {. . II }
\end{aligned}
$$

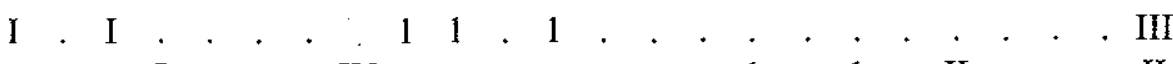

$$
\begin{aligned}
& \text {. . I . . . IV . . . . . . . } 1 \text {. } 1 \text {. II . . . II } \\
& \text { I . III II • . }
\end{aligned}
$$

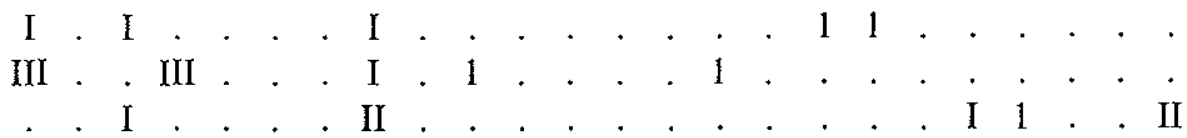

$$
\begin{aligned}
& \text {. I II . . . II . } \\
& \text {. . IV . . . . . . . . } 1 \text {. } 2 \text {. } 12 \text {. . . . . }
\end{aligned}
$$

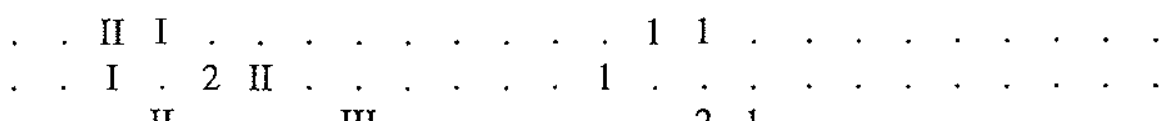

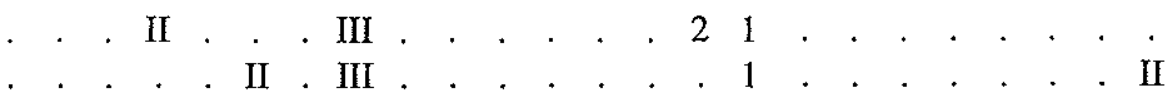

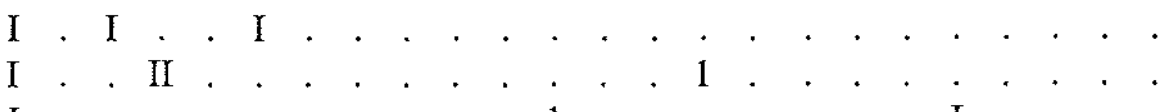

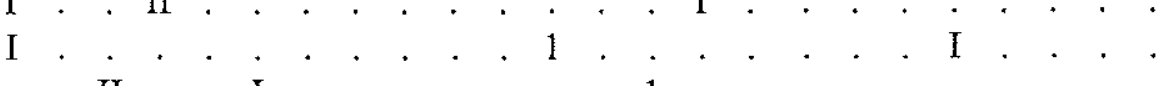

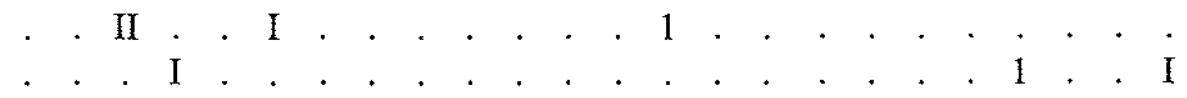

$$
\begin{aligned}
& \text { I. . } 1 \text {. } \\
& \text { II . . . II } \\
& \text { I } 1 \text {. II }
\end{aligned}
$$

Genisto pilosae-Ericetum aragonensis typicum: 1. TARAZONA (1984): tab. 3, rels. 1-4, 7, 9, 10; 2. G. NAVARRO (1986): tab. 42, rels. 1-3; 3. TARAZONA \& ZALDÍVAR 1987, tab. 5, rels. 1-5; rels. 6-7: sub genistetosum anglicae; 4. MEDRANO MORENO (1994): tab. 33, rel. 1; rels. 2-6: sub subass. ericetosum arboreae; 5 . RIVAS-MARTÍNEZ (1962): pag. 111, 3 rels.; 6. RIVAS-MARTÍNEZ (1979): tab. 11, rels. 2-7; 7. Unpublished relevé; 8. MENDIOLA (1983): pag. 256, rels. 4-8; cistetosum laurifolii: 9. TARAZONA (1984): tab. 3, rels. 11-14; 10. G. NAVARRO (1986): tab. 42, rels. 8-9; genistetosum anglicae: 11. TARAZONA (1984): tab. 3, rels. 15, 16;12. G. NAVARRO (1986): tab. 42, rels. 4-7; cytisetosum oromediterranei: 13. TARAZONA (1984): tab. 3, rels. 19, 22; 14. TARAZONA \& ZALDÍvar (1987): tab. 5, rels. 11, 12; 15. MEDRANo MORENo (1994): tab. 33, rels. 7-9; 16. MENDIOLA (1983): pag. 256, rels. 1-3; daboecietosum cantabricae: 17. TARAZONA (1984): 237, tab. 3, rels. 23-25; 18. MEDRANO MORENO (1994): tab. 33, rels. 10, 11; pterospartetosum tridentatae: 19. G. NAVARRO (1986): tab. 42, rels. 10-15. Calluno vulgaris-Genistetum occidentalis typicum: 20. G. NAVARRO (1989): tab. 14, rels. 1-6; ericetosum aragonenis: 21 . G. NAVARRO (1989): tab. 14, rels. 7-10; 22. TÜXEN \& OBERDORFER (1958): tab. 71, I rel.: sub Erica aragonensis-Arctostaphylos uva-ursi; genistetosum micranthae: 23. G. NAVARRO (1989): tab. 14, rels. 11, 12; lavanduletosum pedunculatae: 24. G. NAVARRO (1989): tab. 14, rels. 13-18. 